\title{
Dielectric Constant of Hydrogen-Bonded Liquids
}

\author{
Floyd Buckley
}

\begin{abstract}
A simple extension is presented of Onsager's electrostatic theory of dielectric polarization to take account of the "excess" polarization due to hydrogen bonding. Dielectric data for a number of liquid mixtures are analyzed and the deviations from ideal behavior expressed in terms of an excess polarization. A method is developed for calculating the excess polarization and certain related molecular parameters. Tentative values of these parameters have been determined for some particularly simple structures.
\end{abstract}

\section{Introduction}

The influence of molecular shape on the dielectric constant of polar liquids has been considered in an earlier paper [1]. ${ }^{1}$ The phenomenological theory of Onsager was shown to give a generally satisfactory description of the dielectric constant (1) of pure liquids as a function of the temperature, and (2) of a large class of binary mixtures as a function of the composition. For the restricted class of compounds studied it was found unnecessary to assume specific interactions between nearest neighbors.

The purpose of the present paper is to describe a simple extension of the electrostatic theory to the class of polar liquids that can be considered to possess a pseudo-lattice structure. The order shown by this class is assumed to reflect the existence of strong, specific interactions between neighboring systems. The results of the calculations for a series of related mixtures suggest that the incorporation of these elements of order and interaction into the phenomenological representation of Onsager suffices to give a reasonable agreement with experimental data. A more rigorous analysis along the lines suggested could lead to useful information on the energies of molecular interaction in liquids.

\section{Pure Liquids}

The class of liquids consisting of water and the lower members of the series of normal aliphatic alcohols is considered first to determine the order of magnitude of the polarizability of the hydrogen bonds characteristic of these substances.

\subsection{Model}

The Onsager model of a polar liquid represents a molecule by a cavity with a polarizable point-dipole placed at the center. The volume, shape, polarizability, and intrinsic moment of the cavity are assumed to be the same as those of the molecule. The calculation of the dielectric polarization neglects shortrange forces of interaction between neighbors, and assumes that the influence of long-range forces is fully accounted for by the reaction field produced by the polarization of the continuous dielectric medium surrounding the cavity.

\footnotetext{
1 Figures in brackets indicate the literature references at the end of this paper.
}

In the equivalent representation of a polar liquid which exhibits structure, the molecular cavity of the preceding model is replaced by a representative cluster of molecules that defines a "cell" or "cage" with properties determined, in part, by the local structure. Whereas the intrinsic moment of a cell is taken as characteristic of the molecule within the cell, the "effective" volume, shape, and polarizability of the cell are assumed to depend on the number and polarizability of the bonds (contacts) with nearest neighbors. Each bond is characterized by an interaction energy (strength) and a polarizability that is shared by the cells in contact. In the following discussion it is assumed that the strengths of the bonds are sufficiently weak to permit the dipole to orient freely within its cell. All cooperative effects arising from dipolar interactions are therefore neglected. Actually, it is necessary to assume only that the lifetime of a given molecular cluster is short in comparison with its characteristic relaxation time.

This approach to the problem of the static dielectric constant of hydrogen-bonded liquids is in marked contrast to that of the Kirkwood theory. In the latter, primary attention is focused on the determination of correlation factors and equilibrium constants that characterize the interaction of neighboring dipoles. In the present treatment, attention is centered on the calculation of the polarization of a representative cell (cluster, cavity, molecule) as a function of cell shape, bond polarizabilities, and bond-interaction energies. In view of the quite different information that results from the electrostatic treatment, it was thought worthwhile to investigate this approach as a useful alternative to that of Kirkwood.

\subsection{Polarization}

The calculation of the dielectric polarization follows the procedure of Onsager. The selection of the parameters characteristic of a cell is not unique and at present can be defended only on the grounds of reasonableness. In the present calculations, the intrinsic moment and shape of a cavity are assumed to be "equal" to those of the free molecule, and the remaining parameters, volume and polarizability, are fixed by the conditions:
(a) $\lim \mathscr{P}=\mathscr{P}_{e}($ electronic + atomic + bonding $)$ $\lambda \rightarrow 0$
(b) $\lim _{\lambda \rightarrow \infty} \mathscr{P}=\mathscr{P}_{e}+\mathscr{P}_{0}($ orientation $)$. 
The molar dielectric polarization, $\mathscr{P}$, is given by

$$
\begin{aligned}
& \mathscr{P}=(\epsilon-1) V= \\
& \left\{\frac{R_{e}}{1-\left(R_{e} / V_{c}\right) g k_{R}}+\frac{4 \pi N}{9 k T} \cdot \frac{\mu^{2} k_{c}}{\left(1-\left(R_{e} / V_{c}\right) g k_{R}\right)^{2}}\right\} \frac{9 \epsilon}{2 \epsilon+1},
\end{aligned}
$$

in which $V_{c} / N$ is the volume of a cell, $g, k_{R}$, and $k_{c}$ are the reaction field and cavity parameters [1], $R_{e}$ is the effective molar refraction, and all other symbols have their usual significance.

The only parameter which is assumed to depend on the temperature is the effective refraction, $R_{e}$. This means that if the average contribution of each bond (contact) to the polarizability of the cell is independent of the temperature, then $R_{e}-\left(R_{D}+P_{A}\right)=$ $R_{e}-A$ is proportional to the average number of bonds or "degree of association" of a cell as a function of the temperature. From another point of view, the variation in $R_{e}-A$ is a measure of the fluctuation in the density of polarizability at the boundary of the cell.

The volume of the characteristic cell of a pure liquid should be calculable from a general theory of the liquid state. Although it is not possible at present to do this, any reasonable choice of volume will suffice, because in the simple electrostatic theory the variation of $R_{e}$ with the temperature is probably more significant than the absolute values of $R_{e}$. The principal condition on $V_{c} / N$ is that it must be large enough to enclose the polarizability of the bonds to nearest neighbors.

\subsection{Representation of Experimental Data}

Characteristic cell data are given in table 1 and auxiliary data in table 2 . In table $1, V_{c}$ is the molar cavity volume and, except for water, is taken as the molar volume at the melting point. The quantity $r_{c}$ is the radius of a spherical cavity corresponding to $V_{c} / N ; \mu$, and $e$ are, respectively, the values of the dipole moment and eccentricity assigned to the cavity; and $r_{12}^{m p}$ is the contribution to the polarizability of a cell of each bond between neighboring cells. In table $2, R_{D}$ is the molar refraction for the $\mathrm{Na}-\mathrm{D}$ line; $P_{A}$ is the contribution of atomic polarization to the total distortion polarization $A ; R_{e}^{m p}$ is the value of the effective distortion polarization $R_{e}$ at the melting point; and $\epsilon_{\lambda=0}$ (diel) and $\epsilon_{\lambda=0}$ (disp) are, respectively, the high frequency dielectric constants determined by the conditions given above and from dielectric dispersion data.

The procedure adopted in selecting the values of the cell parameters was the following: It was first assumed that maximum coordination existed at the melting point. The coordination numbers for $\mathrm{H}-\mathrm{O}$. . $\mathrm{H}$ bonds were then taken as 4 for water and 3 for each of the alcohols. It was further assumed that the polarizabilities of all $\mathrm{H}-\mathrm{O}$. . H bonds were the same with a value chosen to match the theory to the data for water. The values of dipole moment and eccentricity given in table 1 for water were assigned to the Onsager cavity and the cavity

\begin{tabular}{|c|c|c|c|c|c|}
\hline Substance & $V_{c}$ & $r_{c}$ & $\mu_{D}$ & $e_{\text {oblate }}$ & $r^{m p}$ \\
\hline $\begin{array}{l}\mathrm{HOH} \\
\mathrm{CH}_{3} \mathrm{OH} \\
\mathrm{C}_{2} \mathrm{H}_{5} \mathrm{OH}_{2} \\
n-\mathrm{C}_{3} \mathrm{H}_{7} \mathrm{OH} \\
n-\mathrm{C}_{4} \mathrm{H}_{9} \mathrm{OH}\end{array}$ & $\begin{array}{l}c m^{3} \\
40.3 \\
34.2 \\
51.0 \\
68.0 \\
85.0\end{array}$ & $\begin{array}{l}\text { A } \\
\text { 2. } 52 \\
\text { 2. } 39 \\
\text { 2. } 72 \\
\text { 3. } 00 \\
\text { 3. } 23\end{array}$ & $\begin{array}{c}1.85 \\
1.70 \\
1.70 \\
1.70 \\
\mathrm{a}(1.67)\end{array}$ & $\begin{array}{l}0.80 \\
.60 \\
.70 \\
.80 \\
.85\end{array}$ & $\begin{array}{c}\text { cm }^{3} \text { bond } \text {-1 }^{-1} \\
\text { mole } \\
2.3 \\
2.3 \\
2.3 \\
2.3 \\
2.3 \\
2.3\end{array}$ \\
\hline $\begin{array}{l}i-\mathrm{C}_{3} \mathrm{H}_{7} \mathrm{OH} \\
t-\mathrm{C}_{4} \mathrm{H}_{9} \mathrm{OH}\end{array}$ & $\begin{array}{l}68.0 \\
95.0\end{array}$ & $\begin{array}{l}\text { 3. } 00 \\
\text { 3. } 35\end{array}$ & $\begin{array}{l}1.70 \\
1.70\end{array}$ & $\begin{array}{l}.80 \\
.50\end{array}$ & $\begin{array}{l}2.3 \\
2.3\end{array}$ \\
\hline
\end{tabular}

TaBle 1. Characteristic cell data

a Quantities in parentheses are estimated.

\begin{tabular}{|c|c|c|c|c|c|c|}
\hline Substance & $\mathrm{mp}$ & $R_{D}$ & $R_{e}^{m p}$ & $\begin{array}{c}\mathrm{a} A=R_{D} \\
+P_{A}\end{array}$ & $\epsilon_{\lambda=0}^{20^{\circ}}($ diel $)$ & $\epsilon_{\lambda=0}^{20 \circ}($ disp $)$ \\
\hline $\begin{array}{l}\mathrm{HOH} \\
\mathrm{CH}_{3} \mathrm{OH} \\
\mathrm{C}_{2} \mathrm{H}_{5} \mathrm{OH} \\
n-\mathrm{C}_{3} \mathrm{H}_{7} \mathrm{OH} \\
n-\mathrm{C}_{4} \mathrm{H}_{9} \mathrm{OH}_{2}\end{array}$ & $\begin{array}{r}{ }^{\circ} \mathrm{C} \\
0 \\
-97.8 \\
-117.3 \\
-127 . \\
-89.8\end{array}$ & $\begin{array}{l}c m^{3} \\
3.67 \\
8.2 \\
12.8 \\
17.7 \\
22.1\end{array}$ & $\begin{array}{l}\mathrm{cm}^{3} \\
13.1 \\
15.5 \\
22.5 \\
27.1 \\
32.1\end{array}$ & $\begin{array}{r}c m^{3} \\
4.0 \\
8.7 \\
15.6 \\
20.2 \\
25.2\end{array}$ & $\begin{array}{l}\text { 5. } 3_{2} \\
\text { 3. } 1_{3} \\
2.9_{3} \\
2.77 \\
2.6_{3}\end{array}$ & $\begin{array}{l}5.2 \\
5.9 \\
3.8 \\
2.7 \\
3.2\end{array}$ \\
\hline $\begin{array}{l}i-\mathrm{C}_{3} \mathrm{H}_{7} \mathrm{OH} \\
t-\mathrm{C}_{4} \mathrm{H}_{9} \mathrm{OH}_{\ldots}\end{array}$ & $\begin{array}{r}-89.5 \\
25.5\end{array}$ & $\begin{array}{c}17.5 \\
\mathrm{~b}(22.1)\end{array}$ & $\begin{array}{l}27.1 \\
34.5\end{array}$ & $\begin{array}{c}20.2 \\
\mathrm{~b}(27.6)\end{array}$ & $\begin{array}{l}\text { 2. } 6_{1} \\
2.6_{1} 1^{\circ}\end{array}$ & $\begin{array}{l}\mathrm{b}(3.2) \\
\mathrm{b}(2.8)^{250}\end{array}$ \\
\hline
\end{tabular}

Table 2. Auxiliary data

a Estimates from dielectric data on gases.

b Quantities in parentheses are estimated.

volume and effective distortion polarization were determined to fit the dielectric data at the melting point. These data were $\epsilon_{\lambda=\infty}=88.0$ and $\epsilon_{\lambda=0}=5.5$. The cell parameters are sensitive to the choice of values for $\epsilon_{\lambda=\infty}$ and $\epsilon_{\lambda=0}$, but any reasonable selection near the values adopted will suffice for an exploratory study.

For the alcohols, the molar cavity volume $V_{c}$ was estimated as the molar volume at the melting point, the dipole moments given in table 1 were assigned, the values of $R_{e}^{m p}=A+3 \times 2.23^{2}$ were calculated, and the eccentricities were then determined to fit the dielectric data. This choice of cavity volume was made because of the relatively large uncertainty in the values of $\epsilon_{\lambda=0}$. There is the additional restriction that this volume must be at least equal to the molar volume.

\subsection{Water}

The available data for liquid water extend from the freezing temperature to the critical temperature and cover a range in density of approximately 1 to 0.5 $\mathrm{g} / \mathrm{cm}^{3}[2,3]$. Recent data on superheated steam at temperatures slightly above the critical temperature extend over a range in density of 0.50 to $0.10 \mathrm{~g} / \mathrm{cm}^{3}$ [4].

The variation of the dielectric polarization with temperature (or density) is completely accounted for by the variation in the effective molar refraction of a representative cell shown in figures 1 and 2 . This dependence of $R_{e}$ on the temperature (or density) can also be translated into a dependence of the "degree of association" on the temperature if the

${ }^{2}$ Values used in the computations deviated slightly from the rounded number 2.3 . 
fraction associated is defined by:

$$
f=\frac{R_{e}-A}{R_{e}^{m p}-A} .
$$

The dependence of $f$ on the temperature is graphically represented in figure 4. An alternative view is that the average coordination number remains constant and the change in $R_{e}$ reflects a corresponding change in the interaction energies and bond polarizabilities between nearest neighbors. This view is adopted in the treatment of mixtures.

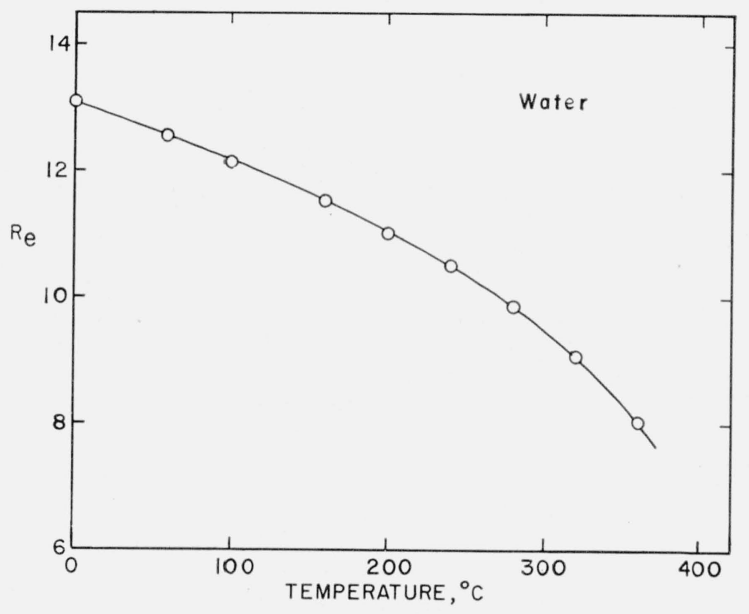

Figure 1. Variation of the effective molar refraction of water from the melting point to the critical temperature.

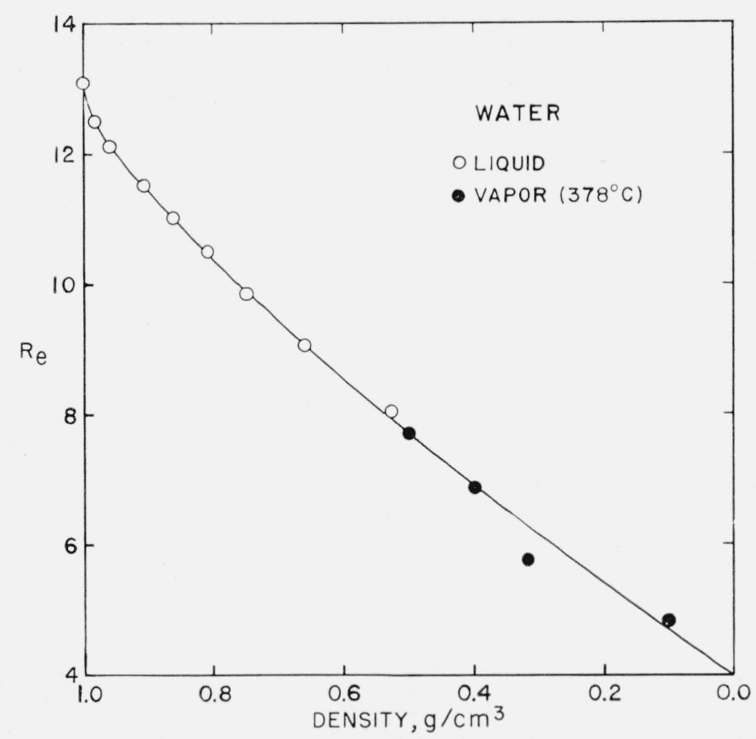

Figure 2. Dependence of the effective molar refraction of water on density.

\subsection{Alcohols}

Data over a wide temperature range are available for only a few of the simpler aliphatic alcohols. Cell characteristics and associated data are given in tables 1 and 2, and the polarizabilities adjusted to fit the polarization data are represented in figure 3. The fraction associated as a function of the temperature is shown in figure 4 .

The strong similarity in dielectric behavior within this class of compounds (including water) is striking, if not entirely unexpected. The anomalous behavior of $t$-butylalcohol, if real, is noteworthy.

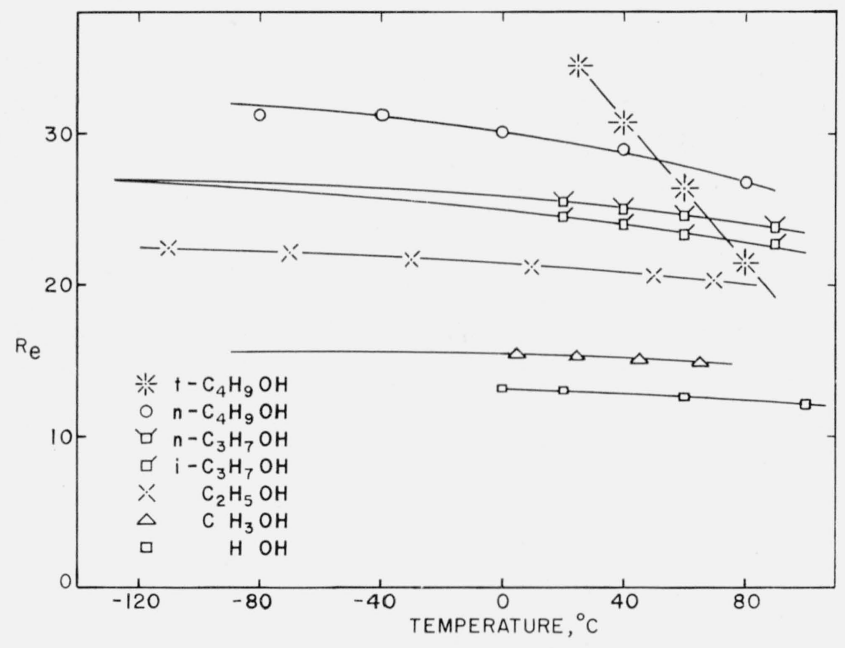

Figure 3. Dependence of the effective molar refraction on temperature.

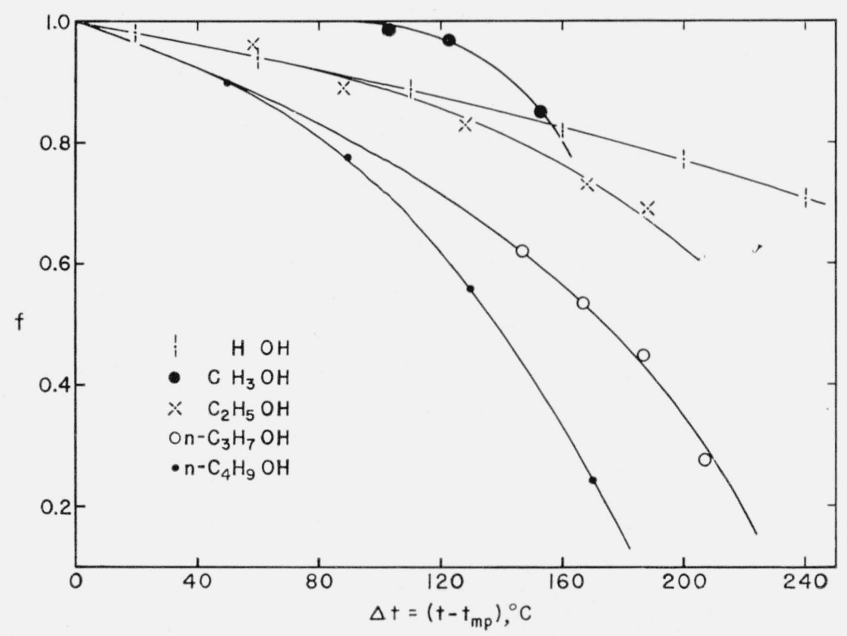

Figure 4. Dependence of the degree of association on the temperature interval above the melting point. 


\section{Mixtures}

\subsection{Preliminary Remarks}

The objective is to express the dielectric polarization of an Onsager spheroidal cavity containing a polarizable point-dipole in terms of an associated orientational partition function.

According to electrostatic theory, the field acting on the dipole is

$$
\vec{E}=\vec{E}_{c}+\vec{E}_{R} \quad \vec{E}_{R}=g \cdot \vec{M},
$$

where $\vec{E}_{c}$ is the field produced within the cavity by external sources, and $\vec{E}_{R}$ is the reaction field arising from the polarization of the medium outside the cavity. The total moment of the cavity is $\vec{M}$ and $g$ is a factor that depends on the shape and volume of the cavity, and on the dielectric constant of the surrounding medium.

The total moment $\vec{M}$ can be written

$$
\vec{M}=\vec{\mu}+\alpha \vec{E}_{c||}+\alpha g_{||} \vec{M}_{\|}+\alpha \vec{E}_{c \perp}+\alpha g_{\perp} \vec{M}_{\perp}=\vec{M}_{\| l}+\vec{M}_{\perp},
$$

in which $\alpha$ is the isotropic polarizability, $\vec{\mu}$ is the dipole moment, and the subscripts $\|$, and $\perp$ designate components parallel and perpendicular to $\vec{\mu}$. It follows that

and

$$
\vec{M}_{\|}=\frac{\vec{\mu}}{1-\alpha g_{\|}}+\frac{\alpha \vec{E}_{c}}{1-\alpha g_{\|}}
$$

$$
\vec{M}_{\perp}=\frac{\alpha \vec{E}_{c \perp}}{1-\alpha g_{\perp}} .
$$

The total energy of the cavity is

$$
\mathscr{E}=-\vec{\mu} \cdot \vec{E}-\frac{1}{2} \vec{M}_{\mu=0} \cdot \vec{E}_{c},
$$

where

Hence

$$
\vec{E}=\vec{E}_{c}+g_{\|} \vec{M}_{\|}+g_{\perp} \vec{M}_{\perp}
$$

and

$$
\begin{aligned}
& \vec{\mu} \cdot \vec{E}=\vec{\mu} \cdot \vec{E}_{c}+g_{\| \mu} \overrightarrow{M_{\|}} \\
& =\frac{\mu E_{1} \mathscr{L} \cos \theta}{1-\alpha g_{\|}}+\frac{{ }^{2} g_{\|}}{1-\alpha g_{\|}}
\end{aligned}
$$

$$
\vec{M}_{\mu=0} \cdot \vec{E}_{c}=\frac{\alpha}{1-\alpha g_{\|}}\left(E_{1} \mathscr{L} \cos \theta\right)^{2}+\frac{\alpha}{1-\alpha g_{\perp}}\left(E_{1} \mathscr{M} \sin \theta\right)^{2},
$$

where the components of the field inside the cavity, parrallel andiperpendicular to the axis of the cavity, are

and

$$
E_{c \mid l}=E_{1} \mathscr{L} \cos \theta
$$

$$
E_{c \perp}=E_{1} \mathscr{C} \sin \theta,
$$

and the external field $\vec{E}_{1}$ makes an angle $\theta$ with the axis of the spheroid containing the dipole. The depolarization factors $\mathscr{L}$ and $\mathscr{M}$ are known.
The self-energy of the cavity is

$$
\mathscr{E}_{\mathrm{S}}=\mathscr{E}_{E_{1}=0}
$$

so that the total energy of the cavity produced by the external field is

$\mathscr{E}_{c}=\mathscr{E}-\mathscr{E}_{s}=\frac{-\mu E_{1}}{1-\alpha g_{\|}} \mathscr{L}_{s} \cos \theta-\frac{1}{2} \frac{\alpha}{1-\alpha g_{\|}}$

$$
\left(E_{1} \mathscr{L} \cos \theta\right)^{2}-\frac{1}{2} \frac{\alpha}{1-\alpha g_{\perp}}\left(E_{1} / / \sin \theta\right)^{2} .
$$

The component of the moment of the cavity along $\vec{E}$, is

$M_{E_{1}}=M_{\|} \cos \theta+M_{\perp} \sin \theta$

$$
=\frac{\mu \cos \theta}{1-\alpha g_{\|}}+\frac{\alpha E_{1} \mathscr{L} \cos ^{2} \theta}{1-\alpha g_{\|}}+\frac{\alpha E_{1} \mathscr{M} \sin ^{2} \theta}{1-\alpha g_{\perp}},
$$

and can be written

$$
M_{E_{1}}=-\left(\frac{\partial}{\partial E_{1} \mathscr{L}}+\frac{\partial}{\partial E_{1} \mathscr{M}}\right) \mathscr{E}_{c} \cdot
$$

Hence the average component is

$$
\begin{aligned}
\bar{M}_{E_{1}} & =\left.\int_{0}^{\pi} \frac{1}{2} M_{E_{1}} e^{-\mathscr{C} c / k T} \sin \theta d \theta\right|_{0} ^{\pi} \frac{1}{2} e^{-\mathscr{E} / k T} \sin \theta d \theta \\
& =k T\left(\frac{\partial}{\partial E_{1} \mathscr{L}}+\frac{\partial}{\partial E_{1} \mathscr{C l}}\right) \ln Q_{c},
\end{aligned}
$$

where

$$
Q_{c}=\int_{0}^{\pi} \frac{1}{2} e^{-\mathscr{E} c / k T} \sin \theta d \theta
$$

is the orientational partition function of the cavity associated with the external field $\vec{E}_{1}$.

The partition function $Q_{c}$ can be written to a sufficient approximation as

$$
\begin{aligned}
&\left.Q_{c} \cong e^{\frac{1}{2} \alpha\left\{\frac{\left(E_{1} \mathscr{L} \cos \theta\right)^{2}}{1-\alpha g||}+\frac{\left(E_{1} \mathscr{M} \sin \theta\right)^{2}}{1-\alpha g_{\perp}}\right\}}\right\}^{\text {ave }} / k T \\
& \int_{\frac{1}{2} e} \frac{\mu E_{1} \mathscr{L} \cos \theta}{1-\alpha g||} / k T \sin \theta d \theta,
\end{aligned}
$$

and if the usual approximations are made

$\ln Q_{c}=\frac{1}{2} \alpha\left\{\frac{1}{3} \frac{\left(E_{1} \mathscr{L}\right)^{2}}{1-\alpha g_{\|}}+\frac{2}{3} \frac{\left(E_{1} \mathscr{M}\right)^{2}}{1-\alpha g_{\perp}}\right\} / k T+$

Hence

$$
\frac{1}{6} \frac{\mu^{2}\left(E_{1} \mathscr{L}\right)^{2}}{\left(1-\alpha g_{\|}\right)^{2}} \frac{1}{(k T)^{2}}
$$

$$
M_{E 1}=\left[\alpha\left(\frac{1}{3} \frac{\mathscr{L}}{1-\alpha g_{\|}}+\frac{2}{3} \frac{\mathscr{M}}{1-\alpha g_{\perp}}+\frac{1}{3} \frac{\mu^{2} \mathscr{L}}{k T\left(1-\alpha g_{\|}\right)^{2}}\right] E_{1},\right.
$$

which is in agreement with former results [1]. 
The dielectric constant is related to $\bar{M}_{E_{1}}$ by the fundamental relation

$$
\epsilon-1=4 \pi \frac{N}{V} \frac{\bar{M}_{E_{1}}}{E_{1}},
$$

so that the molar polarization becomes [1]

$$
\begin{aligned}
\mathscr{P}=(\epsilon-1) V=4 \pi N\left\{\left(\frac{1}{3} \frac{\alpha \mathscr{L}}{1-\alpha g_{\|}}+\frac{2}{3} \frac{\alpha \mathscr{U}}{1-\alpha g_{\perp}}\right)+\right. & \\
& \left.\frac{1}{3 k T \cdot \frac{\mu^{2} \mathscr{L}}{\left(1-\alpha g_{\| 1}\right)^{2}}}\right\} .
\end{aligned}
$$

The relation of the polarization of an Onsager cell to the orientational partition function $Q_{c}$ is utilized in the following section.

\subsection{Binary Mixtures}

The polarization of an ideal mixture is

$$
\mathscr{P}_{12}=\mathscr{P}_{\mathbf{1}} x_{1}+\mathscr{P}_{2} x_{2}
$$

and for a real mixture

$$
\mathscr{P}_{12}=\mathscr{P}_{1} x_{1}+\mathscr{P}_{2} x_{2}+\Delta \mathscr{P}_{m}
$$

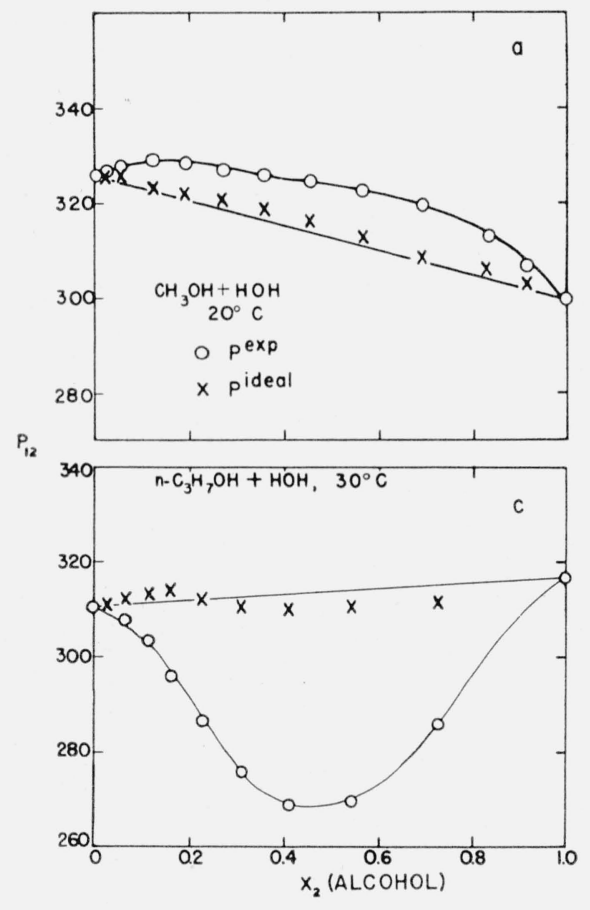

Figure 5. Dielectric polarization (reduced by a factor 1/4.4) for alcoholwater mixtures.

(a) T. T. Jones and R. M. Davies, Phil. Mag. 28, 307 (1939); (b) G. Akerlof, J. Am. Chem. Soc. 54 4125 (1932); (c) G. Akerlof, J. Am. Chem. Soc. $\mathbf{5 4}$ 4125 (1932); (d) G. Akerlof, J. Am. Chem. Soc. 54, 4125 (1932). in which $\mathscr{P}_{1}$ and $\mathscr{P}_{2}$ for the pure components take account of the long-range forces of interaction (Onsager) and the polarization of mixing $\Delta \mathscr{P}_{m}$ allows for the specific interactions of nearest neighbors. An approximate calculation of $\Delta \mathscr{P}_{m}$ can be made in the following way.

Let

$z_{1}=z_{2}=z=$ average number of nearest neighbors to cells (or clusters) of species 1 and 2, respectively,

$n_{11}=$ number of cells of species 1 having only symmetrical 1-1 bonds,

$n_{22}=$ number of cells of species 2 having only symmetrical 2-2 bonds,

$n_{1 j}=$ number of cells of species 1 having $j$ unsymmetrical bonds of type 1-2,

$n_{2 j}=$ number of cells of species 2 having $j$ unsymmetrical bonds of type $2-1$.

Cells of species 1 have a molecule of type 1 at the center of the cell, and those of species 2 have a molecule of type 2 at the center of the cell. Let $w_{11}$, $w_{22}, w_{1 j}$, and $w_{2 j}$ denote the energies of the respective types of cells. Then the total configurational energy of $\mathscr{N}_{1}$ cells of type 1 and $\mathscr{N}_{2}$ cells of type 2 can, to a first approximation, be taken as the sum of an energy of "chemical" binding

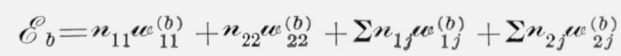
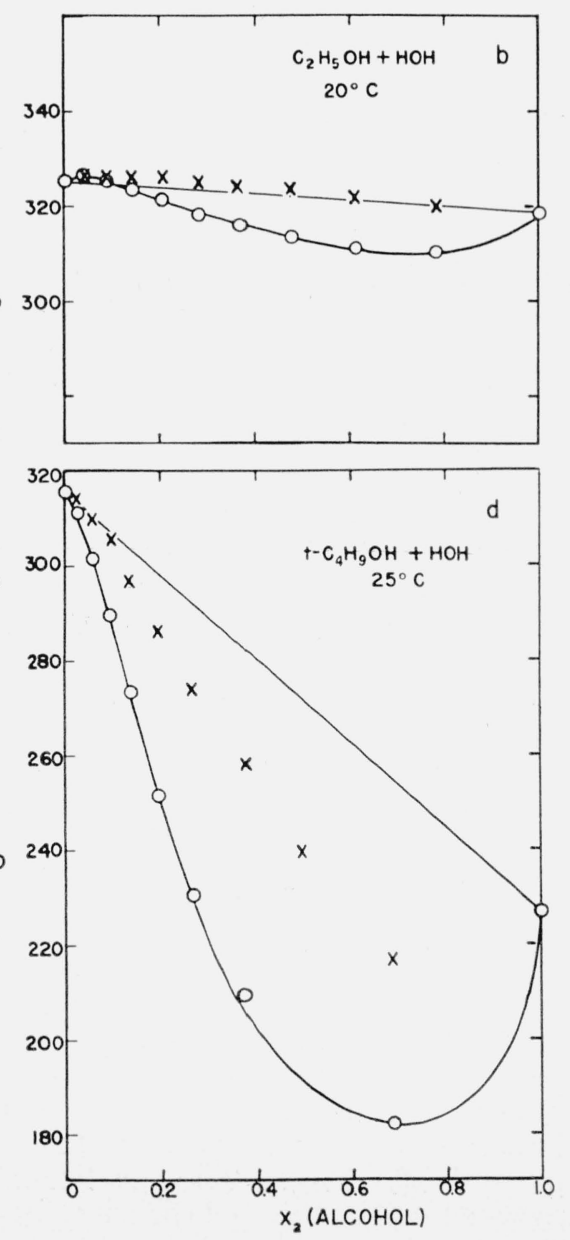


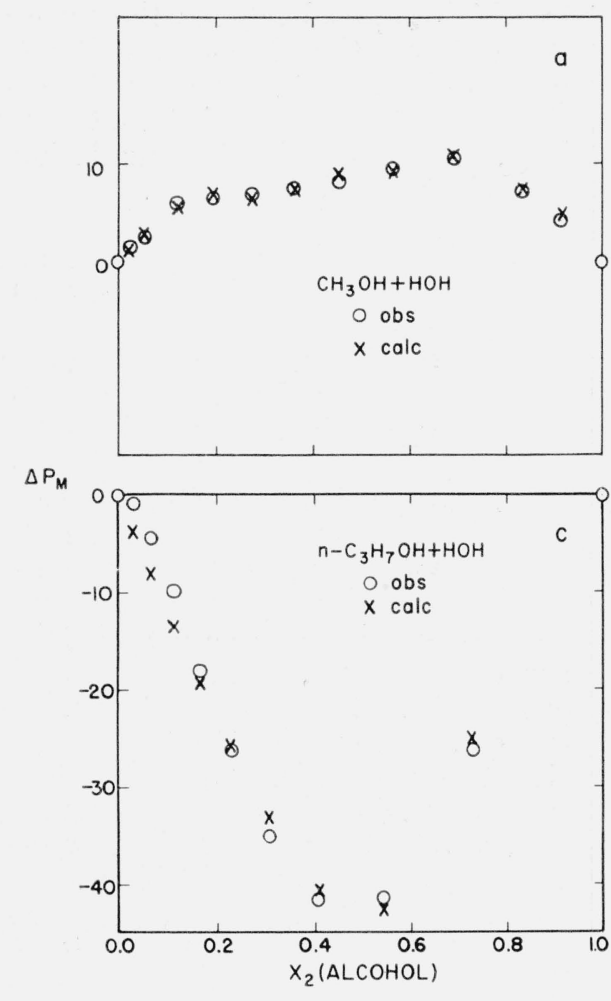

Figure 6. Excess polarization of mixing for alcohol-water mixtures.

and an energy of polarization

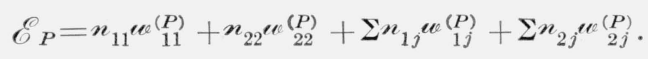

The $N$ 's must satisfy the relations

$$
n_{11}+\Sigma n_{1 j}=\mathscr{N}_{1},
$$

and

$$
n_{22}+\Sigma n_{2 j}=\mathscr{N}_{2} \text {. }
$$

These relations can be used to eliminate $n_{11}$ and $n_{22}$ from the expression for $\mathscr{E}_{P}$ to give

$$
\begin{aligned}
& \mathscr{E}_{P}=\mathscr{N}_{1} w_{11}^{(P)}+\mathscr{N}_{2} \boldsymbol{u}_{22}^{(P)}+\sum n_{1 j}\left(w_{1 j}^{(P)}-\boldsymbol{w}_{11}^{(P)}\right. \\
& +\sum \boldsymbol{x}_{2 j}\left(w_{2 j}^{(P)}-w_{22}^{(P)}\right)=\mathscr{E}_{P}^{\mathscr{O}}+\Delta \mathscr{E}_{P} .
\end{aligned}
$$

The partition function for the system can be written

$$
Q=\Sigma \Sigma Q_{1} \cdot Q_{2} \cdot g\left(\mathscr{N}_{1}, \mathscr{N}_{2}, \boldsymbol{n}_{12}^{*}, \boldsymbol{n}_{21}^{*}\right) e^{-\Delta \mathscr{E}_{P} / k T},
$$

in which $Q_{1}$ and $Q_{2}$ are the partition functions for the species 1 and 2 , respectively, and contain factors which include all contributions to the energy other that $\Delta \mathscr{E}_{P}$. The configurational factor $g$ denotes the number of equivalent configurations for fixed $\mathscr{N}_{1}, \mathscr{N}_{2}, n_{12}^{*}=\Sigma_{n_{1 j}}$, and $x_{21}^{*}=\Sigma_{n_{2 j}}$. The partition function $\Delta Q_{m}$ associated with the polarization of

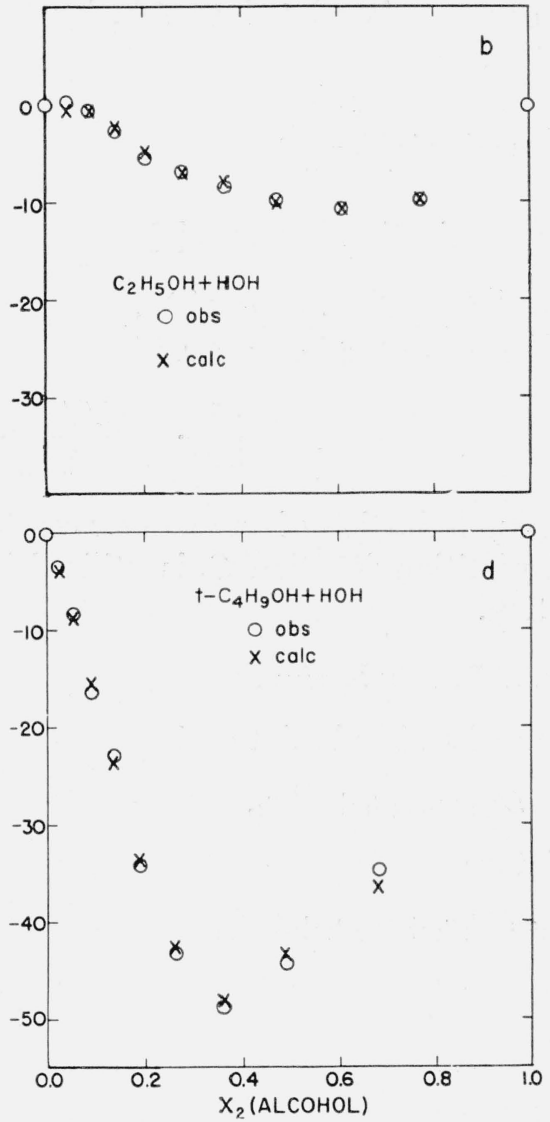

mixing is therefore

$$
\Delta Q_{m}=\Sigma g\left(\mathscr{L}_{1}, \mathscr{N}_{2}, n_{12}^{*}, \underset{n_{21}}{*}\right) \cdot e^{-\Delta \mathscr{E}_{P} / k^{T} T},
$$

and to the zeroth approximation can be replaced by

$$
\Delta Q_{m}^{(0)}=g\left(\mathscr{N}_{1}, \mathscr{N}_{2}, \bar{n}_{12}^{*}, \bar{n}_{21}^{*}\right) e^{-\overline{\Delta \mathscr{C}_{P}} / k T},
$$

in which all variables in $\Delta r_{m}$ have been replaced by average or equilibrium values (denoted by a bar).

The energies of polarization of the Onsager cells appearing in $\overline{\Delta \mathscr{E} P}$ have the same functional form as that evaluated for the Onsager cavity in the case of the pure liquid. Hence the average values $\bar{\kappa}_{12}^{(P)}$ and $\bar{w}_{21}^{(P)}$ appearing in

$$
\overline{\Delta \mathscr{E} P}=\bar{n}_{12}^{*}\left(\overline{\boldsymbol{w}}_{12}^{(P)}-\overline{\boldsymbol{u}}_{11}^{(P)}\right)+\bar{n}_{21}^{*}\left(\overline{\boldsymbol{w}}_{21}^{(P)}-\overline{\boldsymbol{u}}_{22}^{(P)}\right)
$$

can be calculated by the method described under section 3.1, "Preliminary Remarks". The "excess polarization" associated with the mixing process is therefore

$$
\Delta P_{m}=\frac{\bar{n}_{12}^{*}}{\mathscr{N}}(\overline{\mathscr{P}} 12-\overline{\mathscr{P}})+\frac{\bar{n}_{21}^{*}}{\mathscr{N}}\left(\overline{\mathscr{P}}_{21}-\overline{\mathscr{P}}\right),
$$

in which $\overline{\mathscr{P}}$ and $\overline{\mathscr{P}_{21}}$, depend on the composition not only through the reaction field, but also through the "effective polarizability" contributed to a cell by the bonds to its nearest neighbors. 


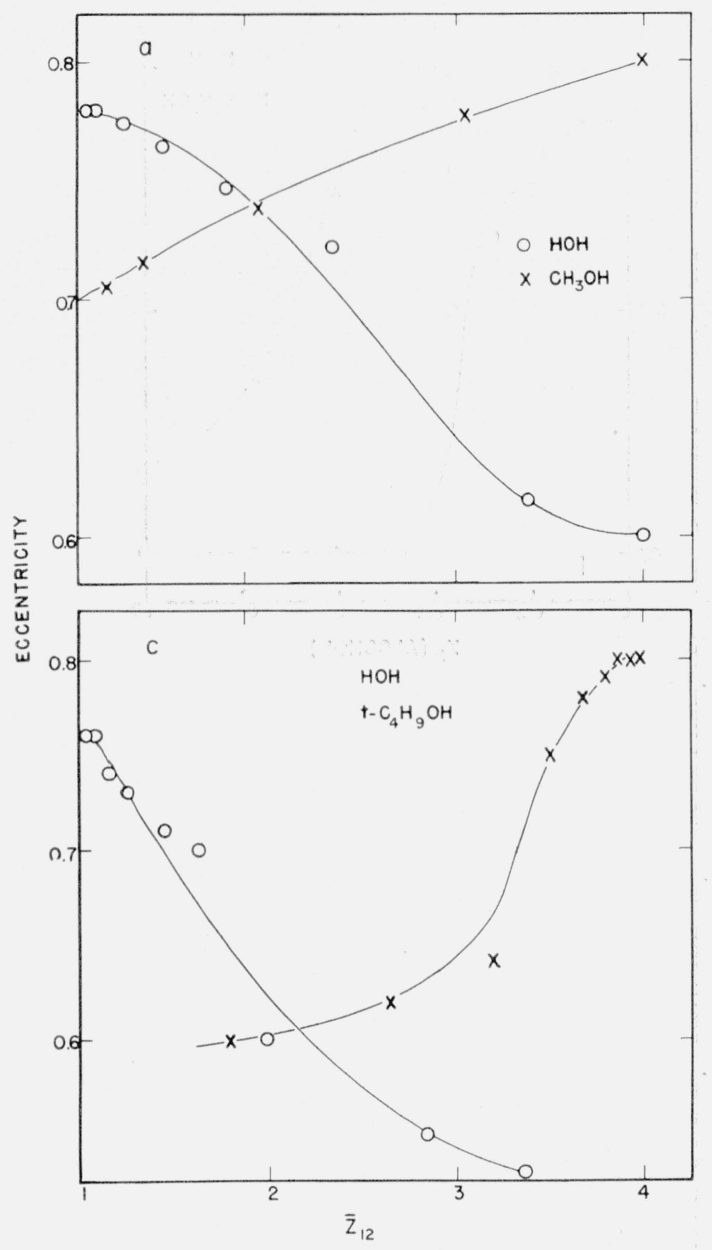

Estimates of $\bar{n}_{12}^{*}$ and $\bar{n}_{21}^{*}$ can be made by using the method introduced by Bethe. The general model of the mixture is that consisting of $\mathscr{N}_{1}$ and $\mathscr{N}_{2}$ molecules of species 1 and 2 , respectively, distributed over a lattice of $\mathscr{N}=\mathscr{N}_{1}+\mathscr{N}_{2}$ sites, in such a way that each site has $z=z_{1}=z_{2}$ nearest neighbors. This shell of nearest neighbors about a central molecule defines the general boundary of an Onsager cell. Its volume, shape, polarizability, and moment need not be specified. The method adopted for determining $\bar{n}_{12}^{*}$ and $\bar{n}_{21}^{*}$ is taken from the work of Rushbrooke [5].

The statistical weight of all configurations with a molecule of species 1 at the center of the complex is

$$
Q_{1}=\lambda_{1} v_{1}\left\{x_{11} \lambda_{1} v_{1}+\epsilon x_{12} \lambda_{2} v_{2}\right\}^{z}=\lambda_{1} v_{1}\left[Q_{1}\right]^{z} ;
$$

in which $\lambda_{1}$ is the absolute activity, $v_{1}$ the local partition function, and the $x_{1 j}$ are bond-interaction factors of the form $e^{-2 w_{1}^{b i} / 2 k T}$. The quantity $\epsilon$ is an "occupational factor" which allows for the interaction of a peripheral molecule of a cell with the surrounding environment. A similar expression is assigned to molecules of species 2 :

$$
O_{2}=\lambda_{2} v_{2}\left\{\epsilon x_{21} \lambda_{1} v_{1}+x_{22} \lambda_{2} v_{2}\right\}^{z}=\lambda_{2} v_{2}\left[Q_{2}\right]^{z} .
$$

The statistical weight of the entire assembly is $Q=Q_{1}+Q_{2}$.

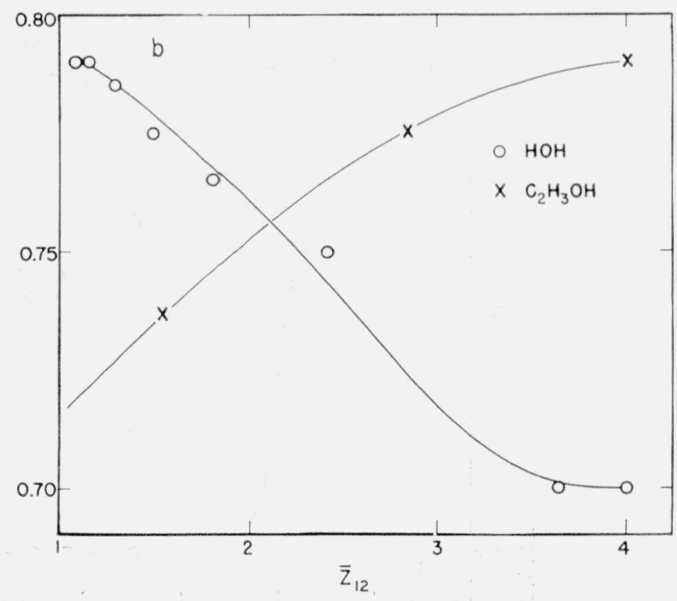

Figure 7. Dependence of the shape of cells of types $n_{12}$ and $n_{21}$ on the mean number of unsymmetrical bonds to a cell.

The equilibrium numbers of molecules of the two species are

and

$$
\mathscr{N}_{1}=\frac{\mathscr{N}}{z+1} \cdot \lambda_{1} \cdot \frac{\partial}{\partial \lambda_{1}} \ln Q_{1}
$$

$$
\mathscr{N}_{2}=\frac{\mathscr{N}}{z+1} \cdot \lambda_{2} \cdot \frac{\partial}{\partial \lambda_{2}} \ln Q_{2}
$$

and the numbers of unsymmetrical bonds $z \overline{\mathscr{X}}$ associated with either of the two types of cells are

$$
\begin{aligned}
\bar{X} & =\frac{1}{2} \frac{\mathscr{N}}{z} \cdot x_{12} \frac{\partial}{\partial x_{12}} \ln Q \\
& =\frac{1}{2} \frac{\mathscr{N}}{z} \cdot x_{21} \frac{\partial}{\partial x_{21}} \ln Q .
\end{aligned}
$$

The condition that a central and peripheral site are equivalent can be expressed by the relation

$$
\lambda_{1} \frac{\partial Q_{1}}{\partial \lambda_{1}}=\lambda_{2} \frac{\partial Q_{2}}{\partial \lambda_{2}}
$$

which leads to

$$
\epsilon=\frac{\left[Q_{1}\right]^{z-1}}{\left[Q_{2}\right]^{z-1}}
$$



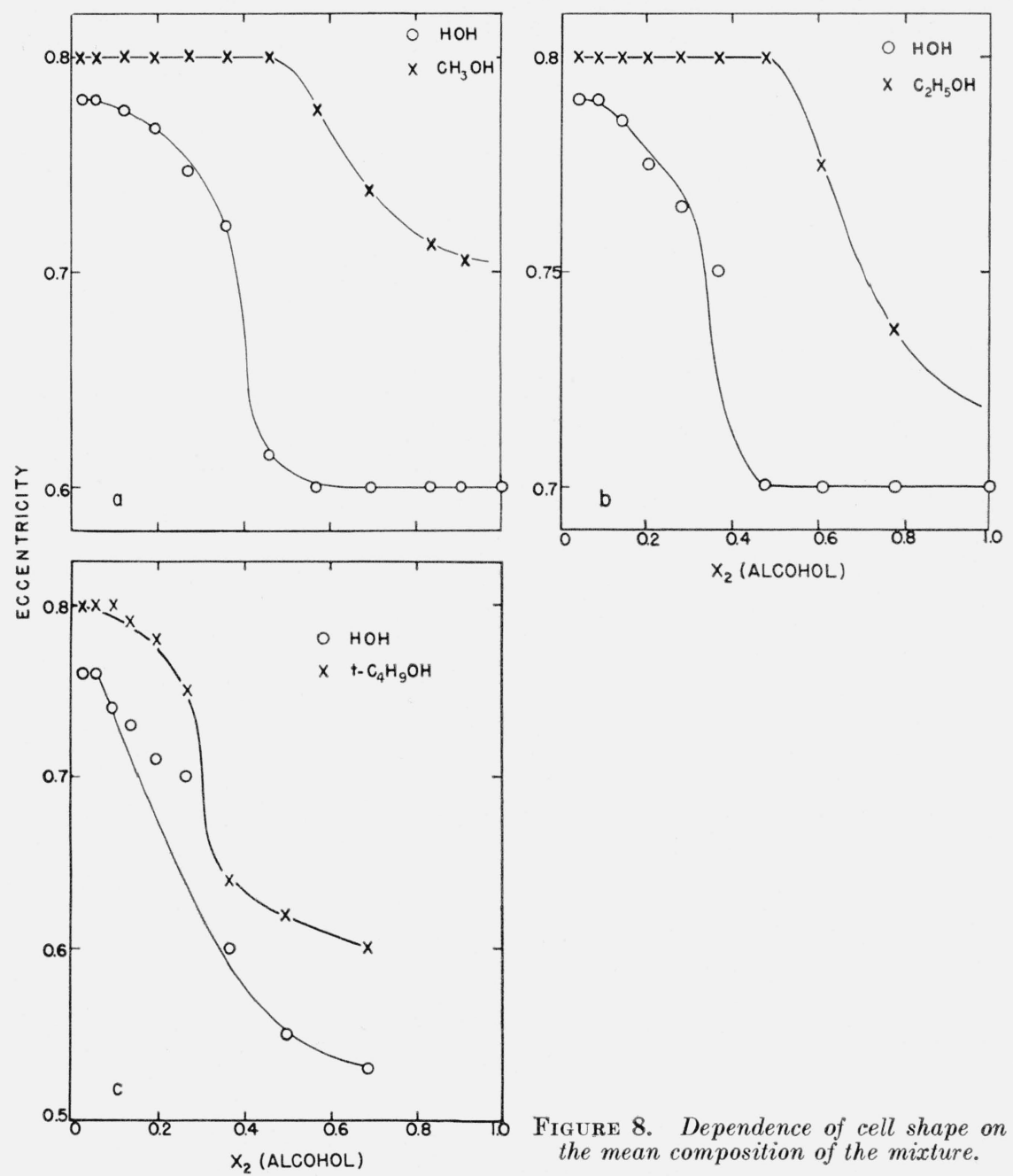

Figure 8. Dependence of cell shape on the mean composition of the mixture.

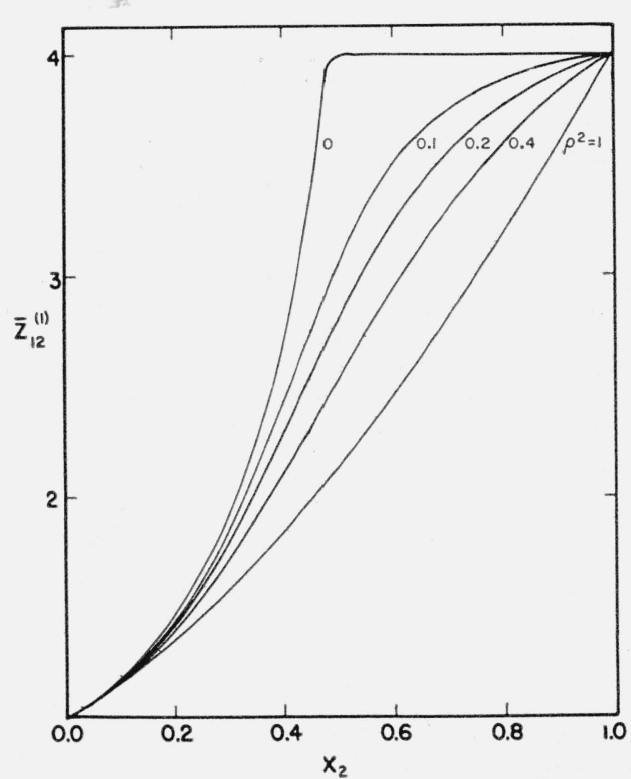

Figure 9. A family of curves representing the dependence of the mean number of unsymmetrical bonds on the mean composition of the mixture and the cell interaction parameter $\rho$. 


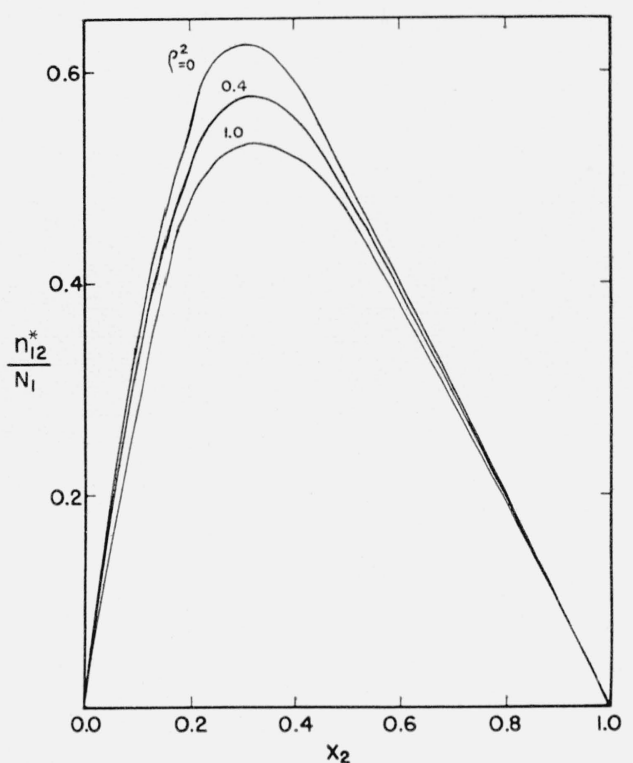

Figure 11. A family of curves representing the dependence of the fraction of all cells of species 1 that have unsymmetrical bonds on the mean composition of the mixture and the interaction parameter $\rho$.

With the aid of this relation it follows that

and

$$
\mathscr{N}_{1}=\mathscr{N} \cdot Q_{1} / Q
$$

$$
\mathscr{N}_{2}=\mathscr{N} \cdot Q_{2} / Q
$$

and $\overline{\mathscr{T}}$ satisfies the equation of a quasi-chemical equilibrium:

$$
\left(\mathscr{N}_{1}-\overline{\mathscr{X}}\right)\left(\mathscr{N}_{1}-\overline{\mathscr{X}}\right)=\overline{\mathscr{X}}^{2} \frac{x_{11} x_{22}}{x_{12}^{2}},
$$

where $x_{12}=x_{21}$. The solution of this equation is

and

$$
\begin{gathered}
\overline{\mathscr{X}}=\mathscr{N} \cdot x_{1} x_{2} \cdot \frac{1}{1+\gamma}, \\
\gamma=\sqrt{1+4 x_{1} x_{2}\left(\rho^{2}-1\right)}
\end{gathered}
$$

$$
\rho^{2}=\frac{x_{11} x_{22}}{x_{12}^{2}}
$$

in which $x_{1}$ and $x_{2}$ are the mole fractions of the two species in the assembly.

The statistical weight of all cells of species 1 which have only symmetrical $1-1$ bonds is $\lambda_{1} v_{1}\left(x_{11} \lambda_{1} v_{1}\right)^{z}$ and the corresponding weight of all cells of species 1 with unsymmetrical $1-2$ bonds is $Q_{1}-\lambda_{1} v_{1}\left(x_{11} \lambda_{1} v_{1}\right)^{2}$. Hence the numbers of the two classes of cells satisfy the relations

and

$$
\bar{x}_{11}^{*}=\frac{\left[Q_{1}\right]^{z}}{\left(x_{11} \lambda_{1} v_{1}\right)^{2}}-1
$$

$$
\bar{n}_{12}^{*}+\bar{n}_{11}==\mathscr{N}_{1}
$$

from which it follows that

$$
\bar{n}_{12}^{*}=\left\{1-\left[1-\frac{\overline{\mathscr{X}}}{\mathscr{N}_{1}}\right]^{z}\right\} \mathscr{N}_{1} .
$$

In a similar way the numbers of cells, $\bar{n}_{12}^{(j)}$, of species 1 which have $j$ unsymmetrical $1-2$ bonds satisfy the equations

$$
\frac{\bar{n}_{12}^{(j)}}{\bar{n}_{11}}=f_{1}^{(j)}
$$

and

so that

$$
\Sigma \bar{n}_{12}^{(j)}=\bar{n}_{12}^{*}
$$

$$
\bar{n}_{12}^{(j)}=\frac{f_{1}^{(j)}}{\Sigma f_{1}^{(j)}} \cdot \bar{n}_{12}^{*}
$$

The $f_{1}^{(j)}$ are the terms of degree $j$ in the polynomial that expresses the ratio of the statistical weight of all cells of species 1 to the weight of all cells of type $\bar{n}_{11}$. This polynomial is:

$$
\begin{aligned}
\left(\frac{x_{11} \lambda_{1} v_{1}+\epsilon x_{12} \lambda_{2} v_{2}}{x_{11} \lambda_{1} v_{1}}\right)^{z} & =\left(1+\frac{\overline{\mathscr{X}}}{\mathcal{N}_{1}-\overline{\mathscr{X}}}\right)^{z} \\
& =\left(1+\frac{x_{2} \cdot 2 / 1+\gamma}{1-x_{2} \cdot 2 / 1+\gamma}\right)^{z} .
\end{aligned}
$$

The fraction of cells of species 1 for the configuration with $j$ unsymmetrical bonds is

$$
\frac{\overline{n_{12}^{(j)}}}{\overline{n_{12}}}=\frac{\left(\frac{x_{2} \cdot 2 / 1+\gamma}{1-x_{2} \cdot 2 / 1+\gamma}\right)^{j}}{\left(1+\frac{x_{2} \cdot 2 / 1+\gamma}{1-x_{2} \cdot 2 / 1+\gamma}\right)^{2}-1}=F_{1}^{(j)} .
$$

Similar expressions hold for cells of species 2 .

Thus:

$$
\bar{n}_{21}^{*}=\left\{1-\left[1-\frac{\overline{\mathscr{X}}}{\mathscr{N}_{2}}\right]^{2}\right\} \mathscr{N}_{2},
$$

and

$$
\frac{\overline{n_{21}^{(j)}}}{\overline{n_{21}^{*}}}=\frac{\left(\frac{x_{1} \cdot 2 / 1+\gamma}{1-x_{1} \cdot 2 / 1+\gamma}\right)^{j}}{\left(1+\frac{x_{1} \cdot 2 / 1+\gamma}{1-x_{1} \cdot 2 / 1+\gamma}\right)^{2}-1}=F_{2}^{(j)} .
$$

The required expression for the excess polarization now becomes

$$
\begin{aligned}
\Delta \mathscr{P}_{m} & =\sum \frac{\overline{n_{12}^{(j)}}}{\mathscr{N}}\left(\overline{\mathscr{P}_{12}^{(j)}}-\overline{\mathscr{P}_{1}}\right)+\sum \frac{\overline{n_{21}^{(j)}}}{\mathscr{N}}\left(\overline{\mathscr{P}_{21}^{(j)}}-\overline{\mathscr{P}_{2}}\right) \\
& =\frac{\overline{n_{12}^{*}}}{\mathscr{N}} \sum F_{1}^{(j)}\left(\overline{\mathscr{P}_{12}^{(j)}}-\overline{\mathscr{P}_{1}}\right)+\frac{\overline{n_{12}^{*}}}{\mathscr{N}} \sum F_{2}^{(j)}\left(\overline{\mathscr{P}_{21}^{(j)}}-\overline{\mathscr{P}_{2}}\right),
\end{aligned}
$$

in which the $\overline{\mathscr{P}_{12}^{(j)}}$ and $\overline{\mathscr{P}_{21}^{(j)}}$ are the polarizations of the Onsager cells corresponding to the $j$-configurations for cells of species 1 and 2 , respectively. In the subsequent analysis of experimental data, the 

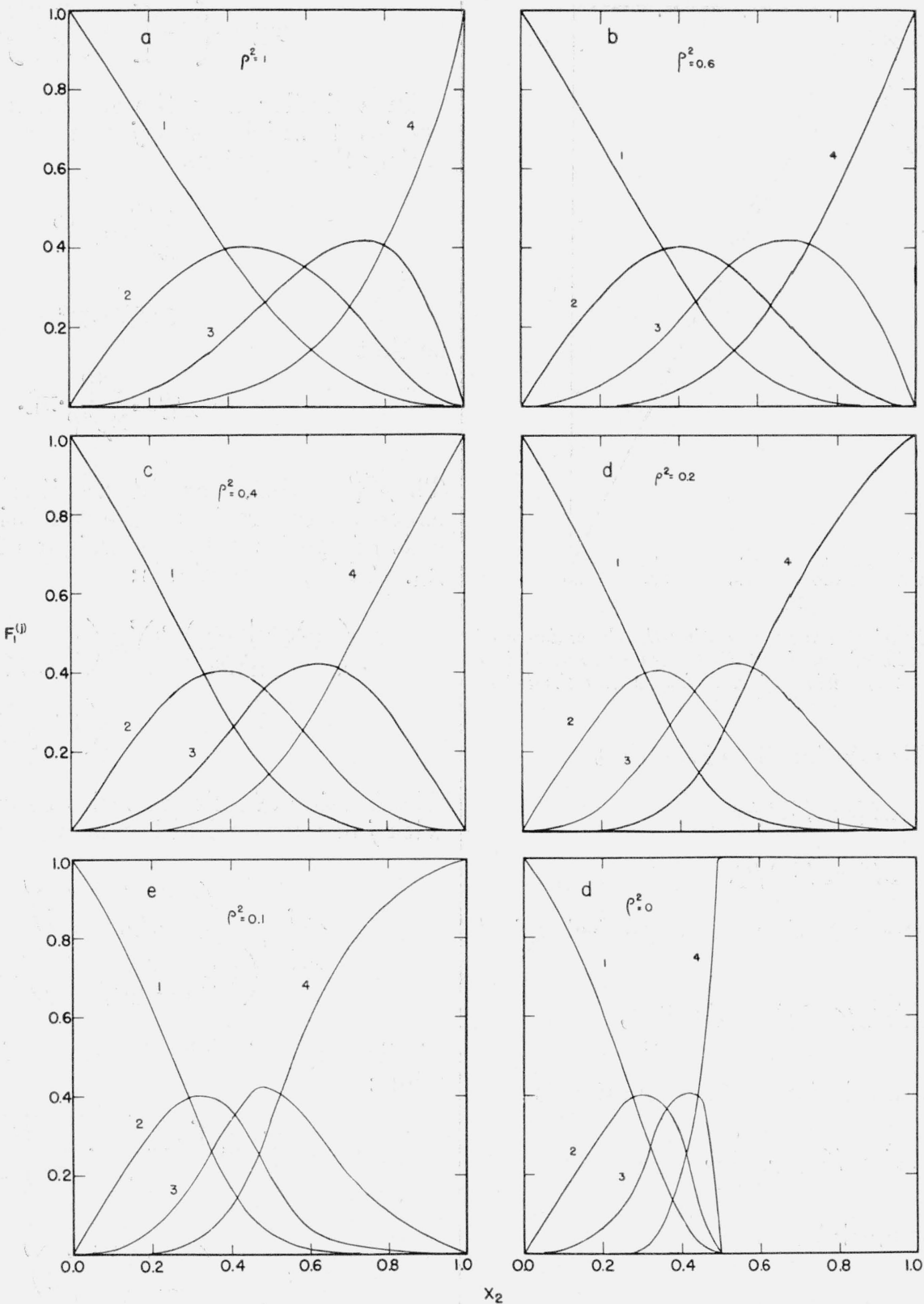

FIGURE 12. A family of curves representing the dependence on composition and interaction parameter $\rho$ of the fractions of cells of species 1 that have 1, 2, 3, 4 unsymmetrical bonds.

quantities $\Sigma F_{1}^{(j)} \overline{\mathscr{P}_{12}^{(j)}}$ and $\Sigma F_{2}^{(j)} \overline{\mathscr{P}_{21}^{(j)}}$ are replaced by $\overline{\overline{\mathscr{P}}_{12}^{(j)}}$ and $\overline{\overline{\mathscr{P}}_{21}^{(j)}}$, which are the polarizations of the corresponding Onsager cells with average configurations: $\bar{z}_{12}=\Sigma j \cdot F_{1}^{(j)}$ and $\bar{z}_{21}=\Sigma j \cdot F_{2}^{(j)}$.

\section{Representation of Experimental Data}

In the preceding discussion it has been assumed that all unsymmetrical bonds binding a cell of either species to its environment are equivalent. This means that $x_{12}=x_{21}$. This assumption obviously expresses only a very crude approximation of the interactions in a cluster. Nevertheless, it is doubtful that the obvious refinement of dividing the bonds to any cell into classes $\alpha, \beta, \ldots$ with approximately equal interaction factors $x_{12}^{(\alpha)}=x_{21}^{(\alpha)} ; x_{12}^{(\beta)}=x_{21}^{(\beta)}$. . . compensates for the added complexity. The simpler picture is retained in the subsequent discussion of special cases.

The analysis of experimental data is reduced to a calculation of the excess polarization of mixing, $\Delta \mathscr{P}_{m}$. In the absence of knowledge of the specific properties of the clusters occurring in a given mixture, such as volume, shape, bond energies, and bond polarizabilities, the calculation becomes one of adjusting the values of selected parameters. This adjustment can be done in a number of ways, but the general procedure adopted was first to choose characteristic bond polarizabilities $r_{12}^{(j)}=r_{21}^{(j)}$ and aver- 


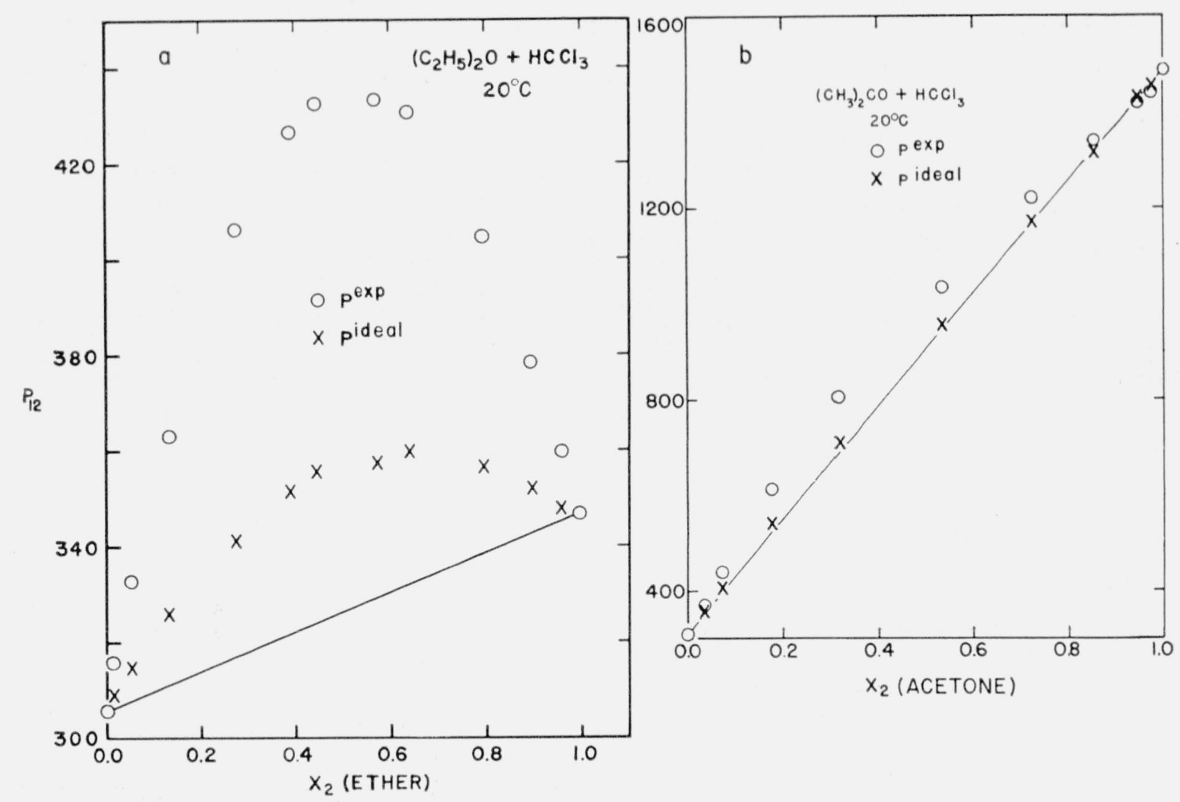

Figure 13. Dielectric polarizations of chloroform-ether and chloroform-acetone mixtures. (a) D. P. Earp and S. Glasstone, J. Chem. Soc. 1935, 1709; (b) D. P. Earp and S. Glasstone, J. Chem. Soc. 1935, 1709.

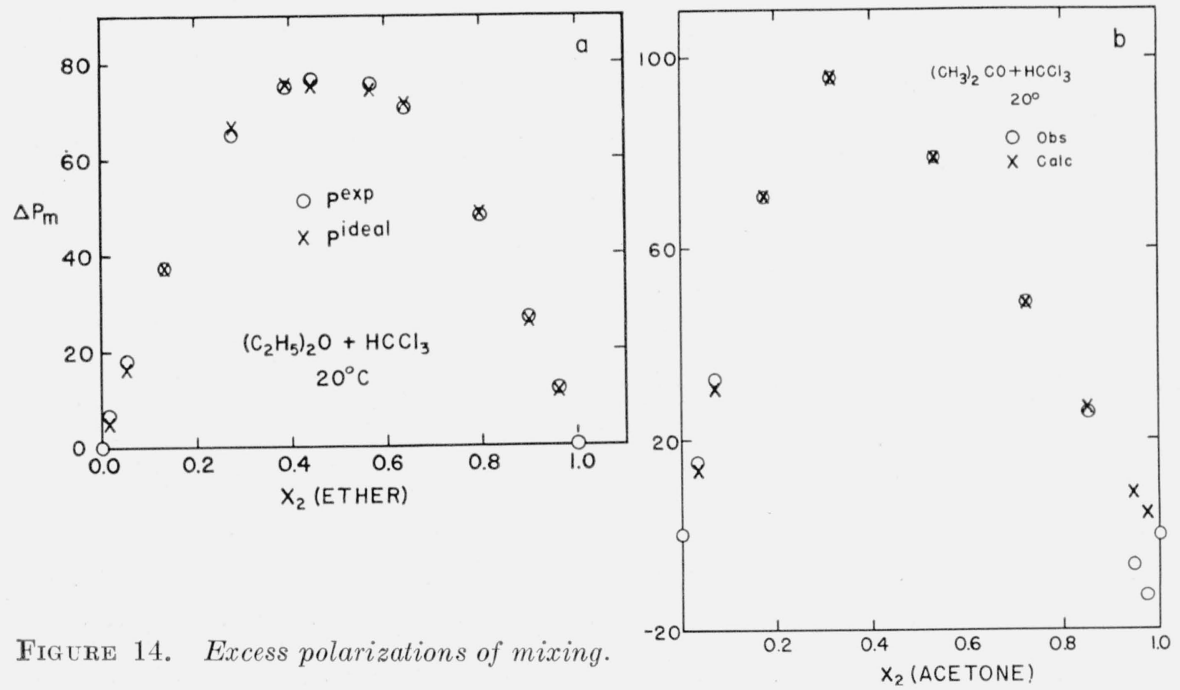

age bond interaction factors $\overline{x_{12}^{(j)}}=\overline{x_{21}^{(j)}}$ and then, if an additional adjustment was necessary, to allow the shapes of the cells to vary with the composition of the boundary of the cell.

For the simplest case all unsymmetrical bonds are equivalent and the eccentricities of cells of types $\bar{n}_{1 j}$ and $\bar{n}_{2 j}$ are those of the pure species. The determination of $\bar{r}_{12}$ is most easily made from the deviations observed at great dilution. At one end of the concentration scale $\bar{z}_{12}=1$ and $\bar{z}_{21}=z$; at the other end $\bar{z}_{12}=z$ and $\bar{z}_{21}=1$. At either end the value of $\Delta \mathscr{P}_{m}$ is very insensitive to the values of the interaction factors $x_{11}, x_{22}$, and $x_{12}$, and these factors can be eliminated by setting them equal to unity (equivalent to setting the bond-interaction energies equal to zero). Moreover, the contribution to $\Delta \mathscr{P}_{m}$ from cells of the component present in small amount becomes negligible at high dilution. Hence, in principle, one calculation suffices to determine $\bar{r}_{12}$. The value of the interaction function $\rho^{2}=x_{11} x_{22} / x_{12}^{2}$ is now fixed by the value of $\Delta \mathscr{P}_{m}$ at any arbitrary concentration, though preferably at the concentration for which $\Delta \mathscr{P}_{m}$ is near a maximum.

In more complex cases, the eccentricities of the cells in the mixtures are functions of composition. Although in these cases the direct calculation of the eccentricities of the various kinds of cells, and of the characteristic mean interaction parameter is, in principle, possible, it is doubtful if the data warrant such a detailed calculation. The values given for these characteristics for any mixture were determined by trial. The method of adjusting these values was as follows: Estimates of the mean eccentricity of cells of type $\bar{n}_{1 j}\left(\right.$ or $\bar{n}_{2 j}$ ) were made on the assumption 

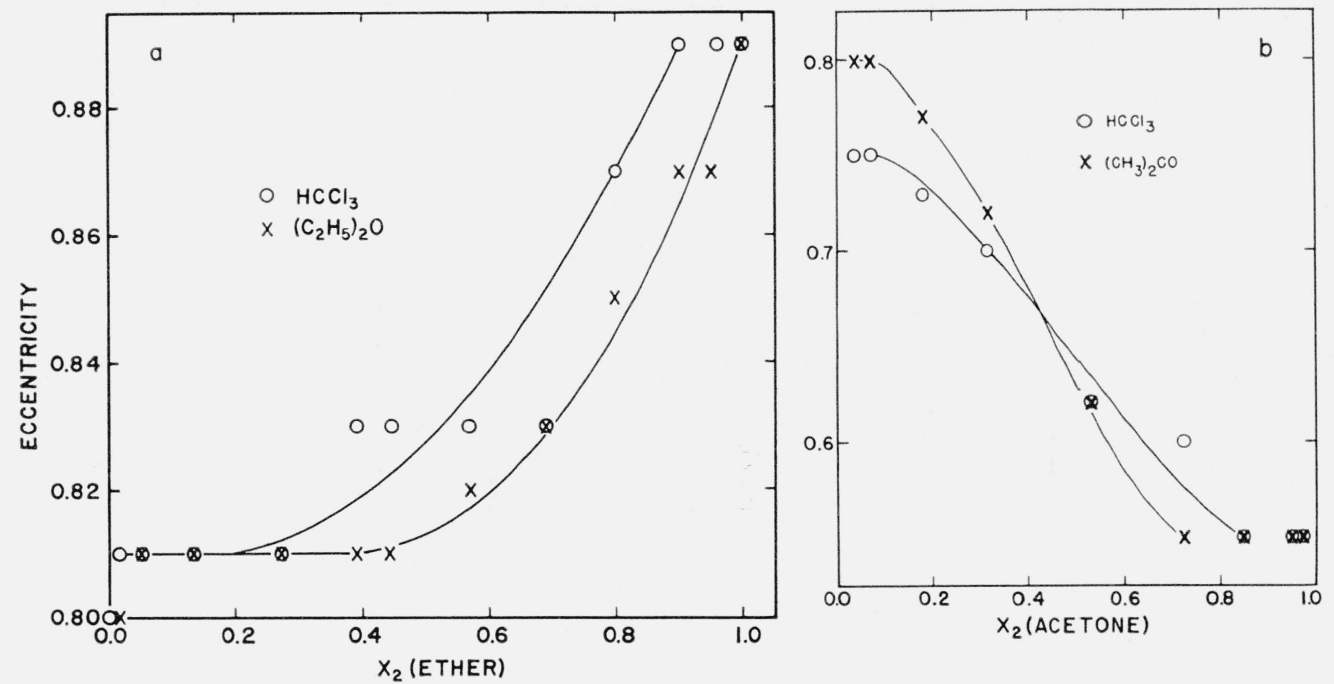

FIgURE 15. Dependence of the shape of cells of types $n_{12}$ and $n_{21}$ on the mean number of unsymmetrical bonds to a cell.
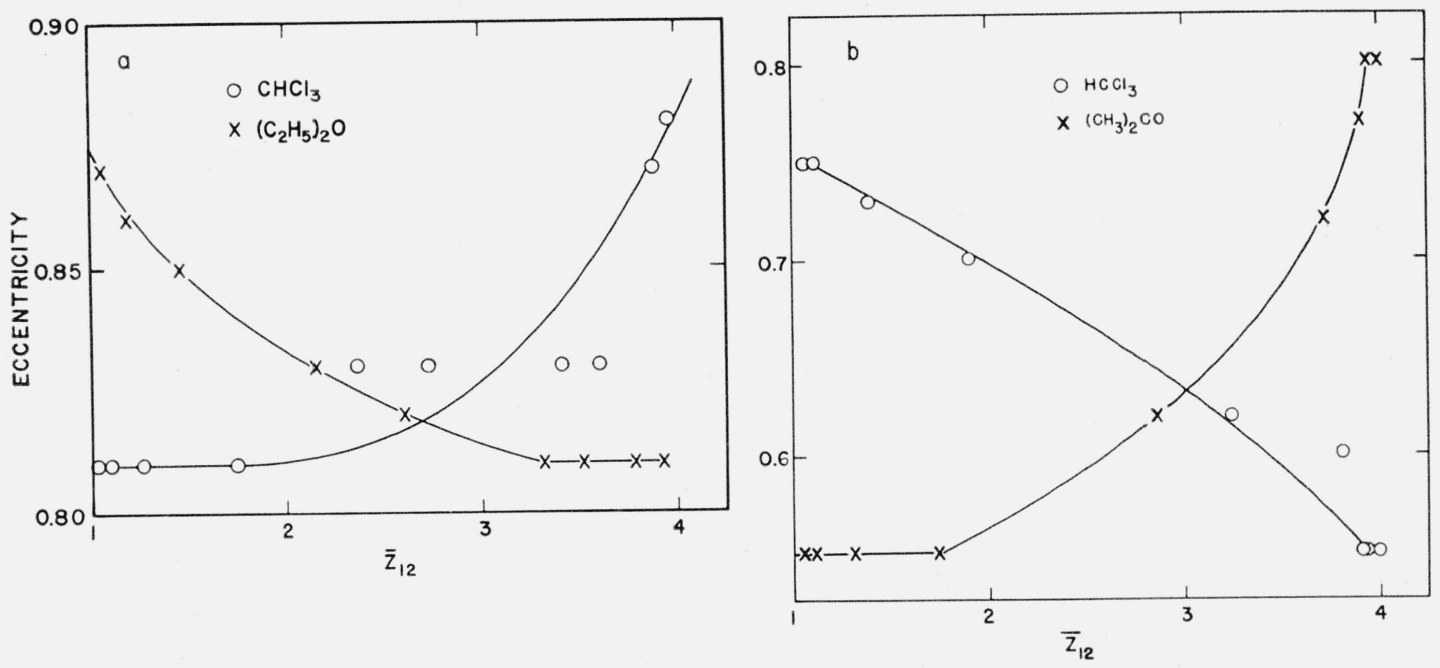

FIgURE 16. Dependence of the shape of cells of types $n_{12}$ and $n_{21}$ on the mean composition of the mixture.

that the eccentricity of any species of cell varied in approximately a linear manner with the number of unsymmetrical bonds. Values were then calculated for a composition near that corresponding to a maximum $\Delta \mathscr{P}_{m}$, and the value of $\rho$ was adjusted to give the observed value of $\Delta \mathscr{P}_{m}$. The mean eccentricities for other concentrations were determined by trial.

In the more general case there are several classes of bonds, each class distinguished by an interaction factor such as $x_{12}^{(\alpha)}$. This distinction of classes is neglected in the calculation of (1) the average number of cells of a given kind, and (2) the average number of unsymmetrical bonds to that cell; but some distinction of classes is recognized in the calculation of the polarizability characteristic of a given cell. In fact each class of bonds is assigned (a) a characteristic bond polarizability, and (b) a position on a scale of relative bond-strengths. The latter permits the determination of the structure of the periphery of a cell for a given $\bar{z}_{12}$ (or $\bar{z}_{21}$ ), and in conjunction with the former, allows the calculation of the effective polarizability of the cell.

\subsection{Mixtures of Water and Alcohols}

The calculations of mean eccentricities for cells of type $\bar{n}_{1 j}$ and $\bar{n}_{2 j}$ were made under the following simplifying assumptions; (a) $z=4$ (one $\mathrm{C}-\mathrm{H}$. . . O and three $\mathrm{O}-\mathrm{H} \ldots \mathrm{O}$ bonds), (b) $x_{12}=x_{21}$, and (c) $\rho^{2}<<1$ (except for $t$-Bu OH). The choice of $z=4$ for the alcohols differs from the value, $z=3$, chosen for the pure liquids and implies that for the purpose of calculating the relative numbers of cells of various types, the interaction parameters for all bonds to the alcohol cells are equal, that is $x_{\mathrm{O}-\mathrm{H}} \ldots \mathrm{O}=$ $x_{\mathrm{C}-\mathrm{H}} \ldots$... This assumption cannot be true, but in view of the crudeness of the model and the gain in simplicity of treatment for the symmetrical case, its adoption is justified for the calculation of effective 

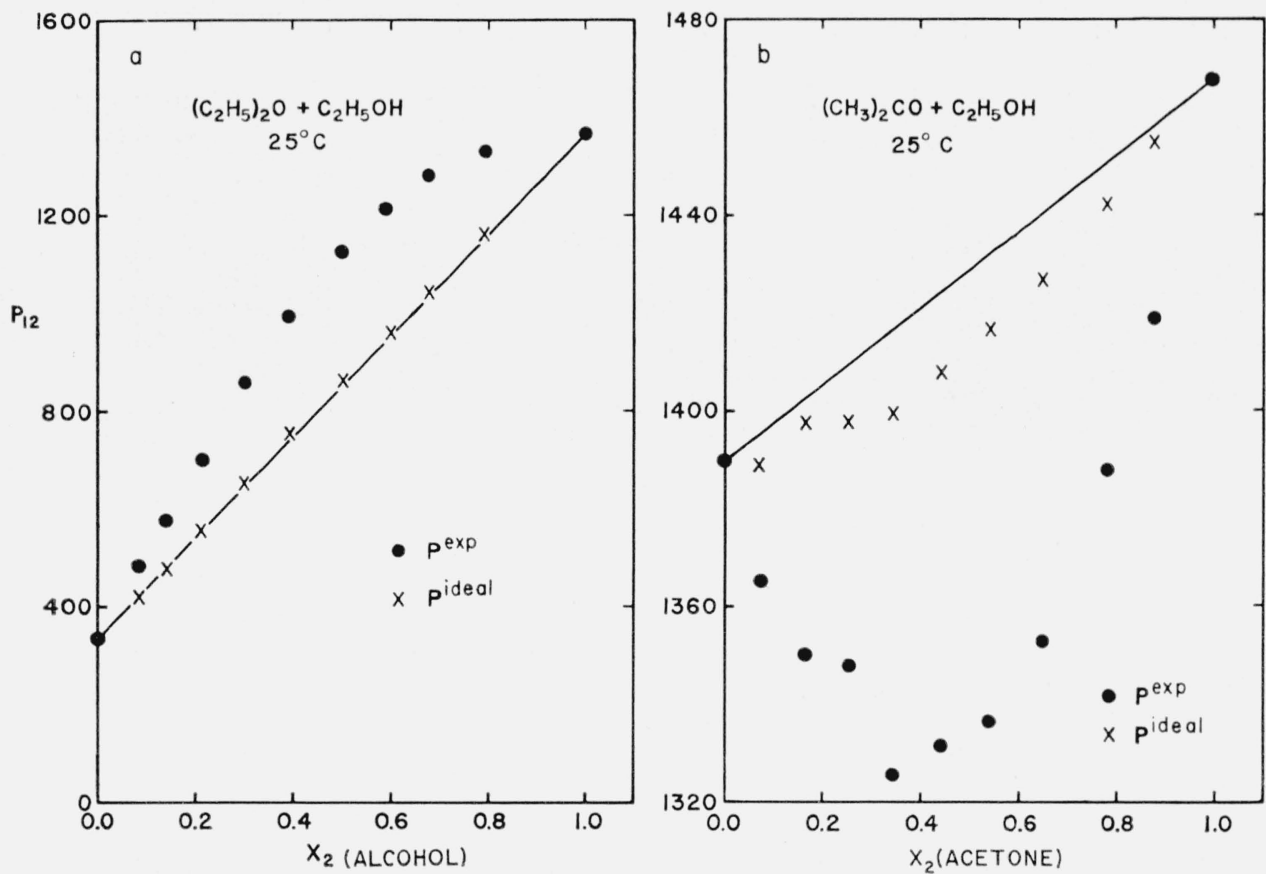

FIGURE 17. Dielectric polarizations for alcohol-ether and alcohol-acetone mixtures. (a) J. Wyman, Jr., J. Am. Chem. Soc. 55, 4116 (1933); (b) W. Graffunder and E. Heyman, Z. Physik. 72, 744 (1931).

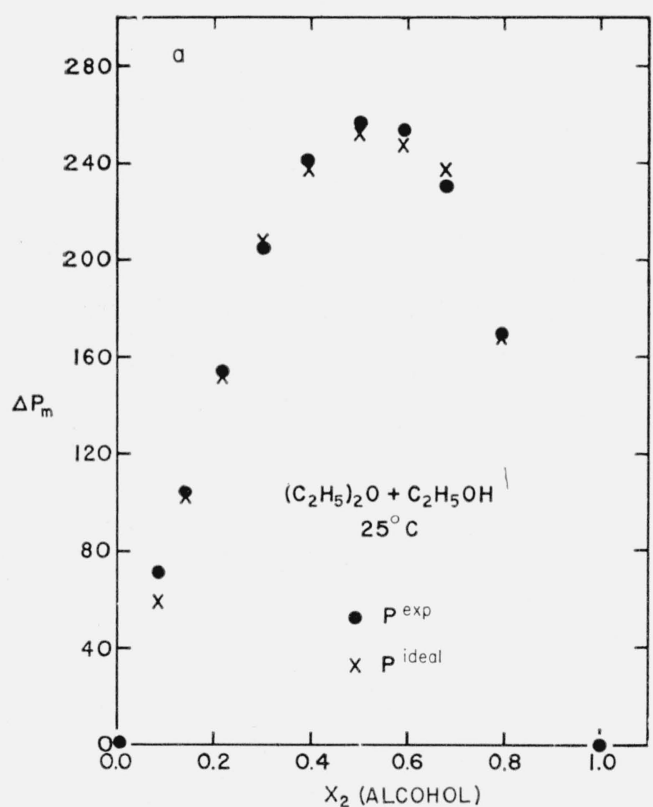

mean shapes. Assumption (c) implies that

$$
-\bar{N} \bar{w}_{\mathrm{cell}}=N\left(-2 \bar{w}_{12}+\bar{w}_{11}+\bar{w}_{22}\right)>>2 \mathrm{RT} .
$$

The calculated eccentricities are relatively insensitive to values of $\rho$ for which $\rho^{2}<<1$ so that for these cases $-N \bar{w}_{\text {cell }}>8 R T\left(4.8 \mathrm{~K}\right.$ cal at $\left.25^{\circ} \mathrm{C}\right)$.

In the calculation of the "effective polarizability" of the average cell of either species, two additional assumptions are made: (d) bonds of type $\mathrm{C}-\mathrm{H}$. . . O do not contribute to the polarizability, and (e) the interaction parameters $x_{\mathrm{O}-\mathrm{H} \ldots \mathrm{O}}$ and $x_{\mathrm{C}-\mathrm{H} \ldots \mathrm{O}}$ are

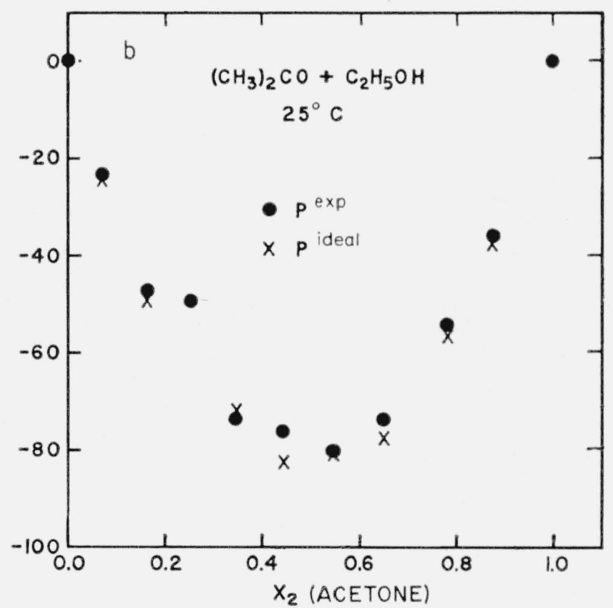

FIGURE 18. Excess polarization of mixing.

"sufficiently" different so that $\mathrm{O}-\mathrm{H}$... O bonds are saturated before $\mathrm{C}-\mathrm{H}$. . . O bonds are formed. Assumptions (b) and (e) are actually contradictory, but in view of the simplification effected they can be taken as compatible for the two types of calculation. Under these restrictions the polarizability of a water cell (species 1 ) is

$$
\begin{aligned}
R_{e}^{\text {eell }} & =R_{D}^{(1)}+\left\{\bar{z}_{12} r_{12}+\left(z-\bar{z}_{12}\right) r_{1}\right\} \\
& =R_{e}^{(1)}-\bar{z}_{12}\left(r_{1}-r_{12}\right) \\
& =R_{e}^{(1)}-\bar{z}_{12} \delta r_{12},
\end{aligned}
$$




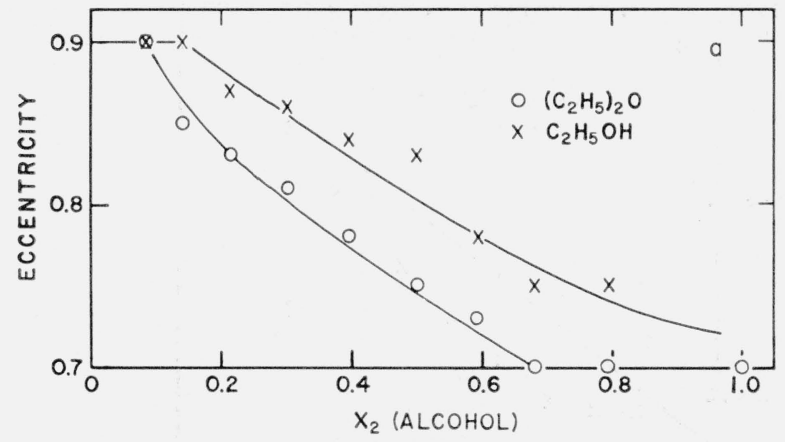

Figure 19. Dependence of the shape of cells of types $n_{12}$ and $n_{21}$ on the mean number of unsymmetrical bonds to a cell.

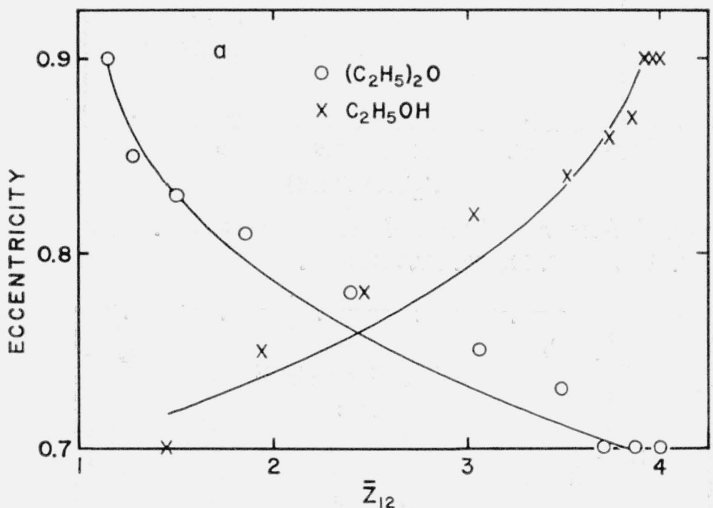

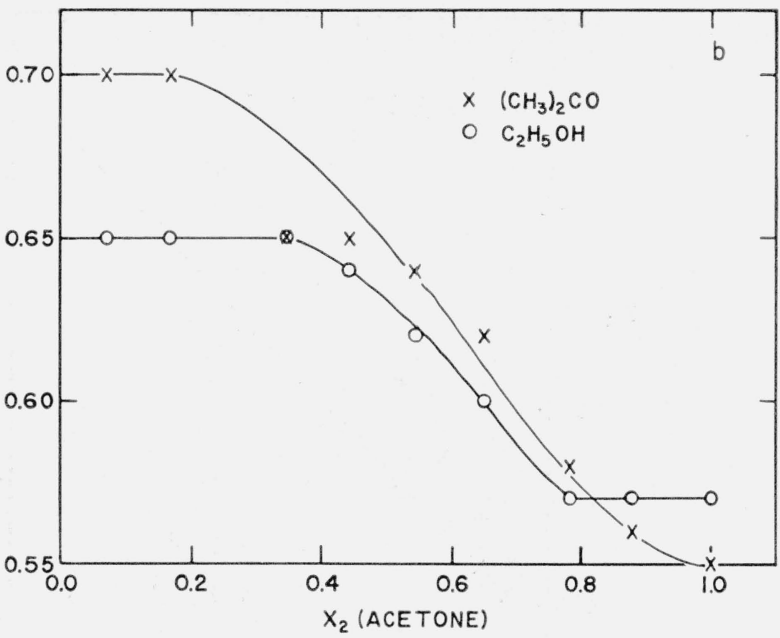

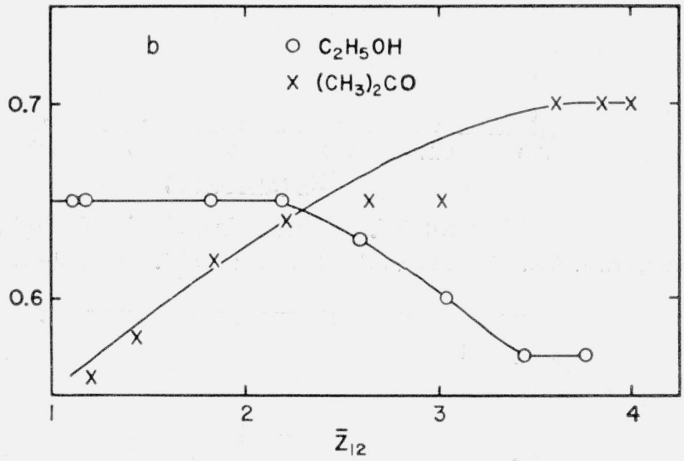

FIGURE 20. Dependence of the shape of cells of types $n_{12}$ and $n_{21}$ on the mean composition of the mixture.

in which $r_{1}$ and $r_{12}$ are the bond polarizabilities of the symmetrical 1-1 and unsymmetrical 1-2 bonds, respectively, and $\bar{z}_{12}$, the mean number of unsymmetrical bonds to a cell, can take the values 1 to 4 . A similar expression holds for the alcohol cells (species 2) with the difference that $z$ is effectively 3 and $\bar{z}_{21}$ can take the values 1 to 3 . The polarizabilities of symmetrical bonds at any temperature were determined from the values of $R_{e}$ given in figure 3 .

Characteristic parameters are given in tables 2 and 3 . The general results of the calculations are summarized in figures 5 and 6 . All molar polarizations have been reduced by an arbitrary factor of $1 / 4.4$. In these figures $\mathscr{P}_{12}^{\text {ideal }}$ is the polarization calculated on the assumption that the shape and polarizability of each cell is that of the pure component, $\mathscr{P}_{12}^{\exp }$ is the experimental value of the polarization, and $\Delta \mathscr{P}_{m}=\mathscr{P}_{12}^{\text {exp }}-\mathscr{P}_{12}^{\text {ideal }}$. The dependence of cell eccentricity on composition is represented in figures 7 and 8.

Curves representing the dependence of $\bar{z}_{12}, \frac{\bar{n}_{12}}{N_{1}}, \frac{\bar{n}_{12}}{N_{1}}$ and $F_{1}^{(j)}$ on $x_{2}$ and $\rho$ are given in figures 9 to 12 . The corresponding curves for component 2 can be obtained by reflecting these curves in the plane $x_{2}=0.5$.
TABle 3. Calculated Parameters

\begin{tabular}{|c|c|c|c|c|}
\hline Substance & $\delta r_{12}$ & $r_{12}$ & $\rho^{2}-1$ & $N \overline{u r}_{\text {cell }}$ \\
\hline $\begin{array}{l}\mathrm{MeOH} \\
\mathrm{H}_{2} \mathrm{O}\end{array}$ & $\begin{array}{c}\mathrm{cm}^{3} \text { bond } \\
\text {-1 } \\
\text { (0) } \text { ole }^{-1} \\
(0)\end{array}$ & $\begin{array}{c}\mathrm{cm}^{3} \text { bond } d^{-1} \text { mole }^{-1} \\
(0.24) \\
(2.24)\end{array}$ & $\begin{array}{l}(0) \\
(0)\end{array}$ & $\begin{array}{c}\mathrm{kcal} / \mathrm{mole} \\
(0) \\
(0)\end{array}$ \\
\hline $\begin{array}{l}\mathrm{EtOH} \\
\mathrm{H}_{2} \mathrm{O}\end{array}$ & $\begin{array}{l}-0.21 \\
-0.62\end{array}$ & 1. 62 & -1 & $<-4.8$ \\
\hline $\begin{array}{l}\mathrm{PrOH} \\
\mathrm{H}_{2} \mathrm{O}\end{array}$ & $\begin{array}{l}-0.28 \\
-0.75\end{array}$ & 1. 48 & -1 & $<-4.8$ \\
\hline $\begin{array}{l}t-\mathrm{BuOH} \\
\mathrm{H}_{2} \mathrm{O}_{\ldots}\end{array}$ & $\begin{array}{l}-1.05 \\
-1.00\end{array}$ & 1. 23 & $\begin{array}{r}-0.7 \\
-\end{array}$ & -1.44 \\
\hline
\end{tabular}

\subsection{Binary Mixtures of Chloroform With Ether and Chloroform With Acetone}

On general physical grounds, both mixtures are expected to show evidence of hydrogen bonding. The data are graphically represented in figure 13 and the agreement between $\Delta \mathscr{P}_{m}{ }^{\text {obs }}$ and $\Delta \mathscr{P}_{m}{ }^{\text {cale }}$ is shown in figure 14. Because of the dissimilarity in molecular constitution and structure, the shape of any cell should depend on the composition of its periphery. The variations of the eccentricities with the average number of unsymmetrical bonds and with the composition of the mixture are shown in figures 15 and 16 . Selected parameters are given in table 4 . 


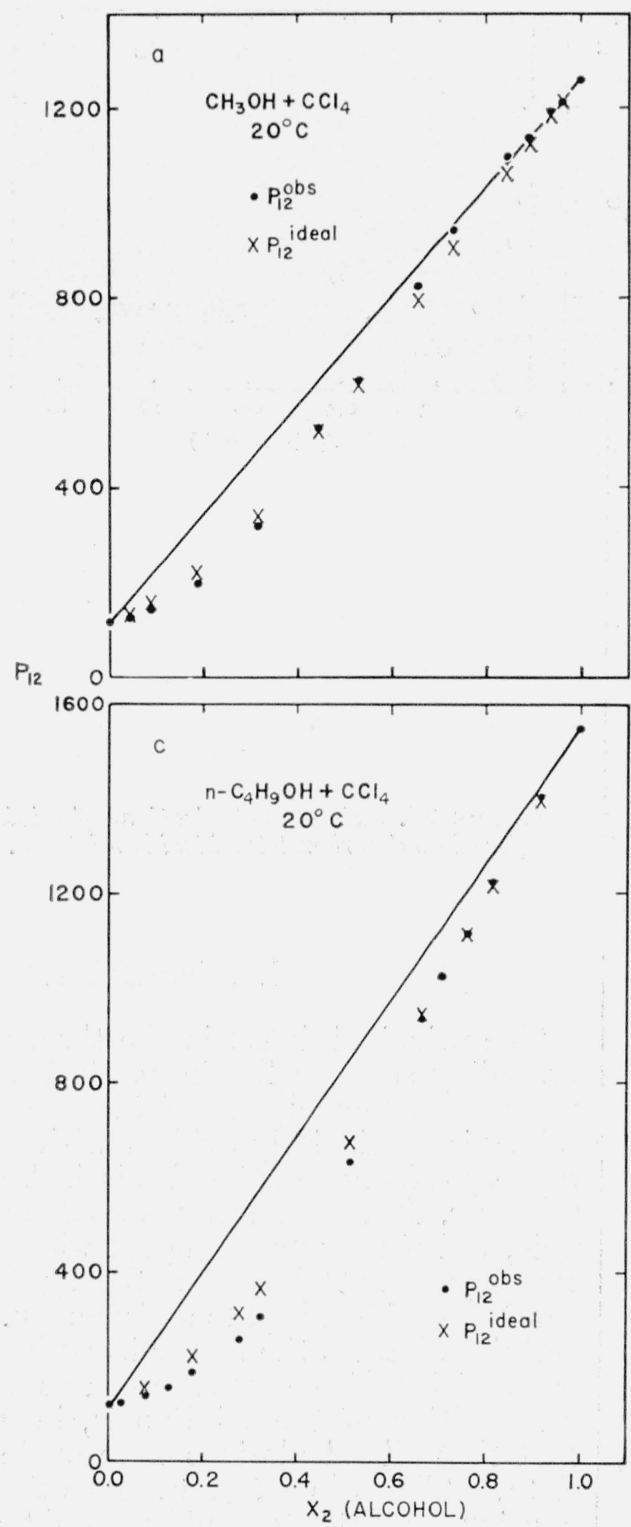

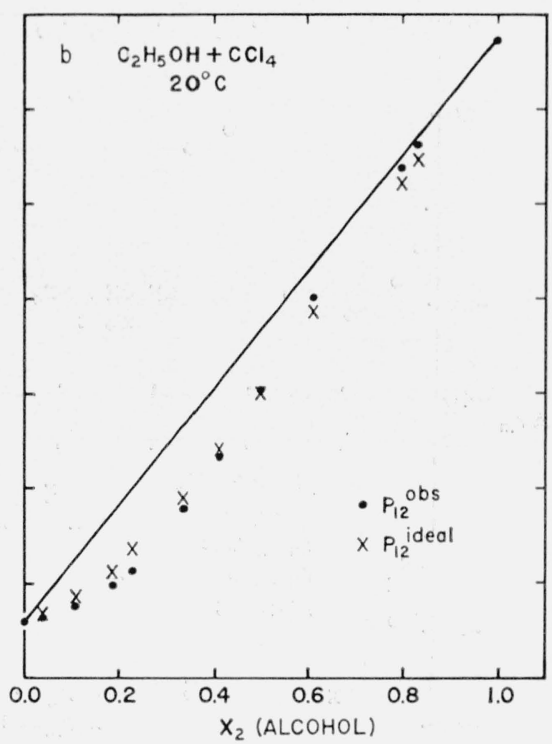

Figure 21. Dielectric polarizations of alcohol-carbon tetrachloride mixtures.

(a), (b), and (c), R. Mecke and A. Reuter, Z.
The behavior of the systems bromoform-ether and bromoform-isopropyl ether parallels that of the chloroform-ether system.

TABLE 4. Calculated parameters

\begin{tabular}{|c|c|c|c|c|}
\hline Substance & $\delta r_{12}$ & $r_{12}$ & $\rho^{2}-1$ & $N \overline{\boldsymbol{e}}_{\text {cel1 }}$ \\
\hline $\begin{array}{l}\text { Chloroform } \\
\text { Ether }\end{array}$ & $\begin{array}{c}\mathrm{cm}^{3} \text { bond } \\
4 \\
4 \\
4\end{array}$ & $\begin{array}{c}\mathrm{cm}^{3} \text { bond } \\
4\end{array}$ & -0.90 & $\begin{array}{c}\mathrm{kcal} / \text { mole } \\
-2.8\end{array}$ \\
\hline $\begin{array}{l}\text { Chloroform } \\
\text { Acetone }\end{array}$ & $\begin{array}{l}1 \\
1\end{array}$ & 1 & -0.90 & -2.8 \\
\hline
\end{tabular}

\subsection{Binary Mixtures of Alcohol With Ether and Alcohol With Acetone}

For these related mixtures the signs of the polarization of mixing are opposite. The data are graph- ically represented in figure 17 and the agreement between $\Delta \mathscr{P}_{m}{ }^{\text {obs }}$ and $\Delta \mathscr{P}_{m}{ }^{\text {eale }}$ is shown in figure 18 . The parameters selected to reproduce this behavior are given in table 5 . In comparison with table 4 these values appear quite reasonable and illustrate the flexibility inherent in the simple electrostatic picture. Variations in the shape of the characteristic cells are shown in figures 19 and 20.

TABle 5. Calculated parameters

\begin{tabular}{|c|c|c|c|c|}
\hline Substance & $\delta r_{12}$ & $r_{12}$ & $\rho^{2}-1$ & $N \bar{\omega}_{\text {cell }}$ \\
\hline $\begin{array}{l}\text { EtOH } \\
\text { Ether }\end{array}$ & $\begin{array}{c}\mathrm{cm}^{36} \text { bond } d^{-1} \text { mole }^{-1} \\
\text { 3. } 00 \\
\text { 4. } 80\end{array}$ & $\begin{array}{c}\mathrm{cm}^{3} \text { bond } d^{-1} \text { mole }^{-1} \\
4.80\end{array}$ & -0.90 & $\begin{array}{c}\mathrm{kcal} / \mathrm{mole} \\
-2.8\end{array}$ \\
\hline $\begin{array}{l}\text { EtOH } \\
\text { Acetone }\end{array}$ & $\begin{array}{r}-0.7 \\
1.1\end{array}$ & 1. 10 & -0.50 & -0.84 \\
\hline
\end{tabular}



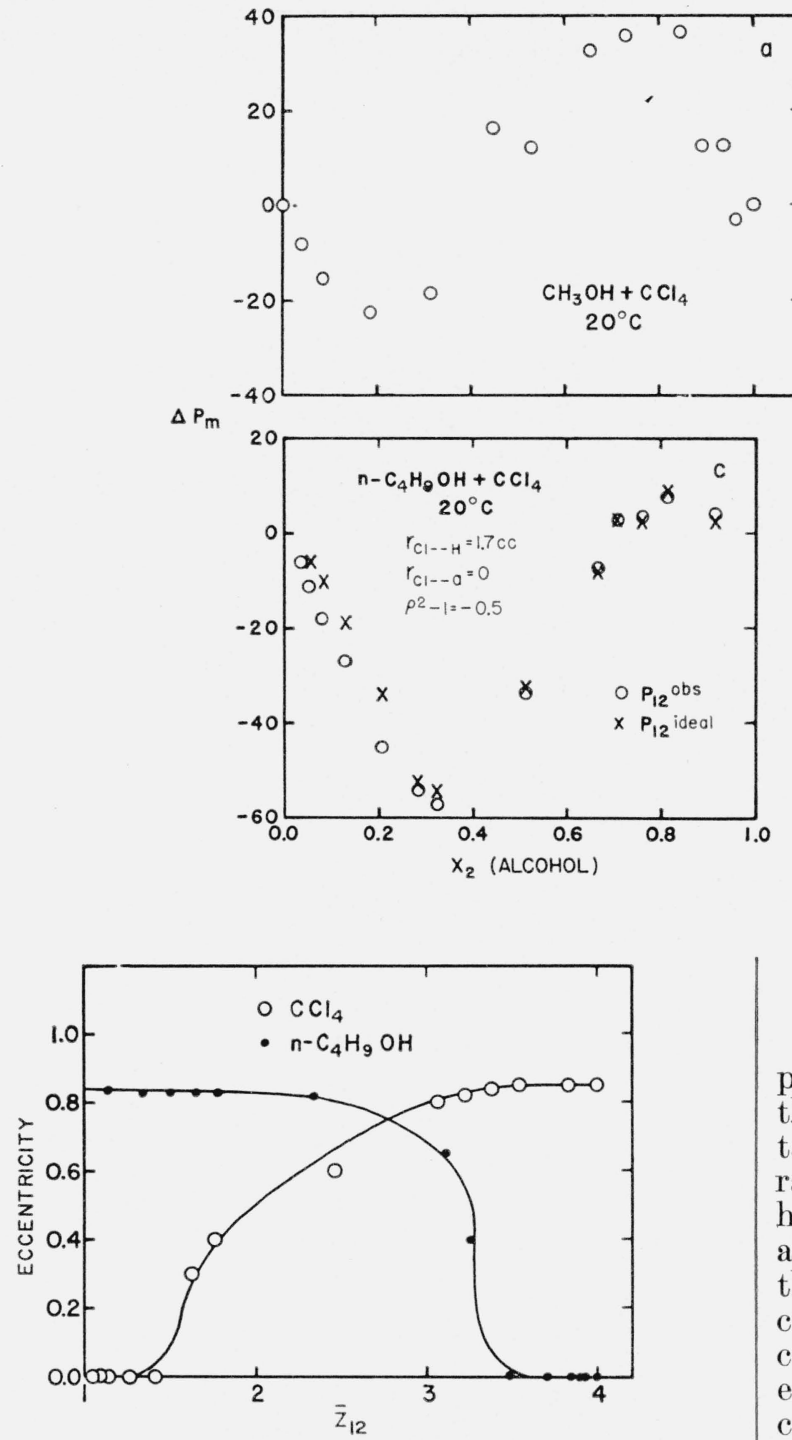

Figure 23. Dependence of the shape of cells of types $n_{12}$ and $n_{21}$ on the mean number of unsymmetrical bonds to a cell.

\subsection{Mixtures of Carbon Tetrachloride and the Simple Alcohols}

The behavior of these systems is shown in figures 21 and 22 . The system $n-\mathrm{C}_{4} \mathrm{H}_{9} \mathrm{OH}-\mathrm{CCl}_{4}$ is the only one treated in detail. Parameters and their dependence on composition are given in figure 23. The general similarity of the remaining systems suggests that the other mixtures are amenable to the same type of treatment.

\subsection{Mixture of Water With Dioxane}

This system could be expected to have a behavior somewhat between the water-alcohol systems and the alcohol-ether system. Its behavior is represented in figure 24 .

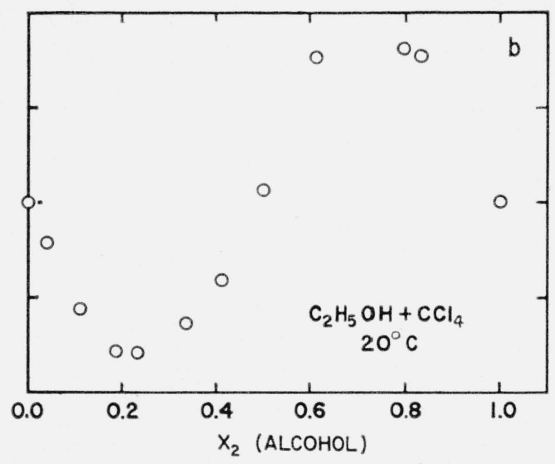

FIGURE 22. Excess polarizations of mixing for alcohol-carbon tetrachloride mixtures.

\section{Discussion}

The principal defect in the theory outlined in the preceding sections lies in the lack of uniqueness of the parameters characterizing a cell. The uncertainty is particularly evident with regard to the parameters of shape and bond polarizability. It appears, however, that the selections made are not unreasonable, and if they are accepted with this reservation, the agreement between theory and experiment can be considered quite satisfactory. In the case of certain mixtures the rather irregular behavior of the eccentricity of the average cell as a function of the composition of the solution is, in part, due to the fact that all calculations were made on the original data. The use of data smoothed to eliminate spurious deviations would undoubtedly improve the regularity in the behavior of this characteristic.

The general utility of the method of analysis described lies in the fact that it offers an independent method for obtaining important information on molecular interactions within liquids. A careful study of a selected series of related mixtures should provide relative values of bond strengths and stabilities of molecular clusters. These quantities may be useful in correlating related macroscopic and molecular properties, such as relative acidity and basicity of groups, mutual solubilities, vapor pressures, and viscostities. Such investigations in which adequate attention has been given to purity of materials and accuracy of measurement are very few.

The bond polarizabilities $r_{12}$, interaction parameters $\rho$, and the predominant features of the curve representing the polarization as a function of the composition are summarized in table 6 . The results are tentative, and since values and trends may 


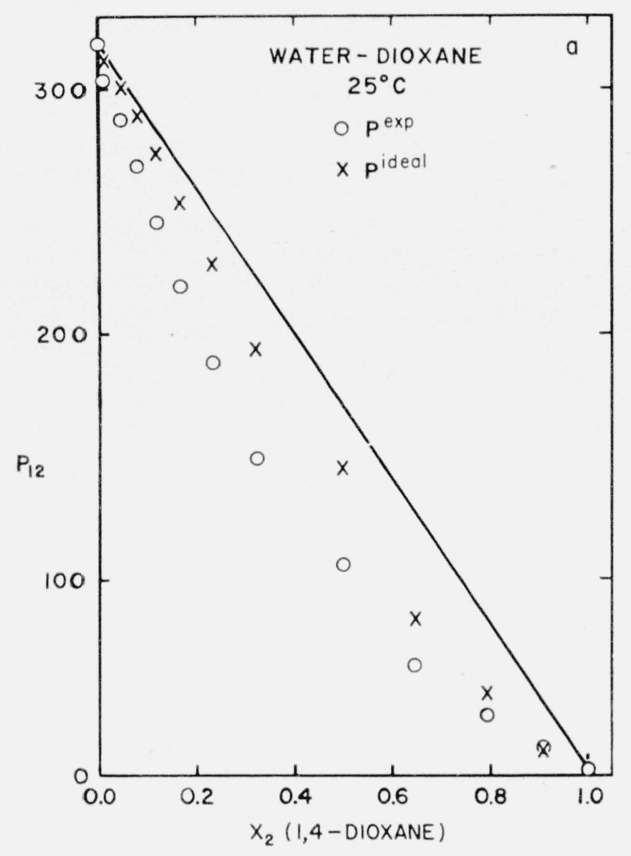

Figure 24. Dioxane-water mixtures.

a, Dielectric polarization (reduced by a factor of $1 / 4.4$ ) (G. A kerlof and O. A. Short, J. Am. Chem. Soc. 58, 1241 (1936)); b, Excess polarization of mixing; $c$, Dependence of the shape of cells of types $\boldsymbol{n}_{12}$ and $\boldsymbol{n}_{21}$ on the mean number of unsymmetrical bonds to a cell; d, Dependence of the shape of cells of types $m 12$ and $\boldsymbol{n}_{21}$ on the mean composition of the mixture.
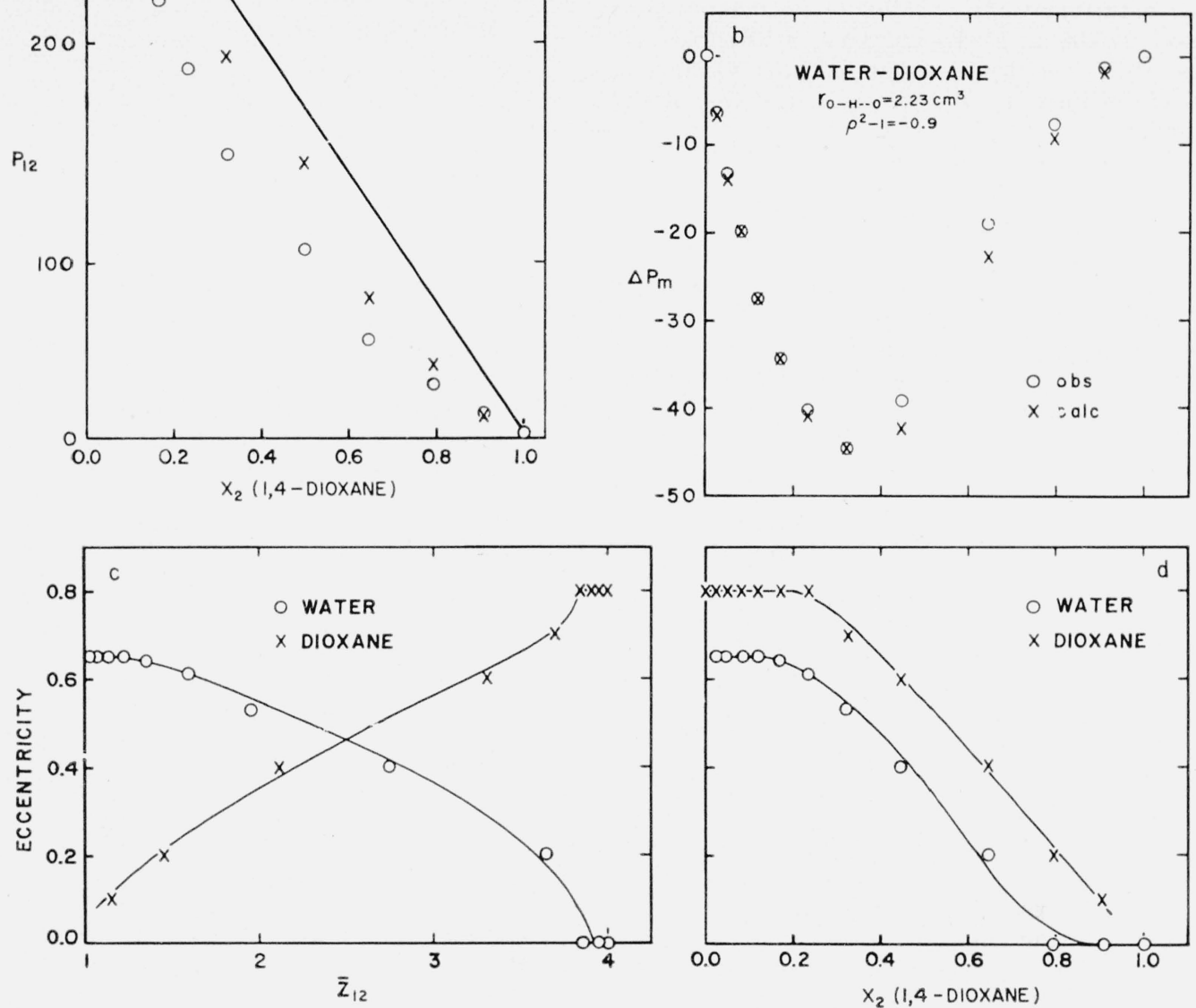

TABLE 6. Summary of cell characteristics

\begin{tabular}{|c|c|c|c|c|}
\hline Type of bond & Mixture & Polarization characteristic & $r_{12}$ & $\rho^{2-1}$ \\
\hline $\mathrm{O}-\mathrm{H} \ldots \mathrm{O}_{\ldots} \ldots$ & $\left\{\begin{array}{l}\text { Dioxane-water } \\
\text { Methyl alcohol-water } \\
\text { Ethyl alcohol-water } \\
n \text {-Propyl alcohol-water } \\
t \text {-Butyl alcohol-water } \\
\text { Ether-ethyl alcohol } \\
\text { Acetone-ethyl alcohol }\end{array}\right.$ & $\begin{array}{l}\text { Minimum } \\
\text { Maximum } \\
\text { Minimum } \\
\text { Minimum } \\
\text { Minimum } \\
\text { Maximum } \\
\text { Minimum }\end{array}$ & $\begin{aligned} & \mathrm{cm}^{3} \text { bond }-1 \\
& \text { mole }^{-1} \\
& \\
& 2.23 \\
& 2.24 \\
& 1.62 \\
& 1.48 \\
& 1.23 \\
& 4.80 \\
& 1.10\end{aligned}$ & $\begin{array}{l}-0.90 \\
0 \\
-1 \\
-1 \\
-0.70 \\
-0.90 \\
-0.50\end{array}$ \\
\hline $\mathrm{O}-\mathrm{H} \ldots \mathrm{Cl}_{\ldots} \ldots$. & t-Butyl alcohol-carbon tetrachloride & Maximum and minimum & 1.7 & -0.50 \\
\hline $\mathrm{C}-\mathrm{H} \ldots \mathrm{O}_{\ldots}$ & $\left\{\begin{array}{l}\text { Ether-chloroform } \\
\text { A cetone-chloroform }\end{array}\right.$ & $\begin{array}{l}\text { Maximum } \\
\text { Maximum }\end{array}$ & $\begin{array}{l}4.0 \\
1.0\end{array}$ & $\begin{array}{l}-0.90 \\
-0.90\end{array}$ \\
\hline
\end{tabular}


be significantly altered by the choice of different models, speculations based on the results obtained for this limited number and variety of systems are probably premature.

It is noticed that the contribution of the $\mathrm{CH} \ldots \mathrm{O}$ bond to the polarizability is considerably larger in the mixtures of ether and chloroform than in those of acetone and chloroform. This large difference indicates either a failure of the rather crude model that has been used in the analysis, or that additional refinements in the treatment must be introduced in order to take adequate account of the specific nature of bond interactions.

Clues to the existence and character of the complexes in mixtures may be found in the excess (static) dielectric polarization, but conclusive evidence should be provided by their dielectric absorption spectra. These data should yield important information on the number, type, and lifetimes of the various complexes which are often assumed to play a significant role in chemical reactions. Data of this kind are not available.

\section{References}

[1] F. Buckley and A. A. Maryott, J. Research NBS 53, 229 (1954) RP2539.

[2] G. C. Akerlof, J. Am. Chem. Soc. 54, 4125 (1932).

[3] G. C. Akerlof and H. I. Oshry, J. Am. Chem. Soc. 92,2844 (1950).

[4] J. K. Fogo, S. W. Benson, and C. S. Copeland, J. Chem. Phys. 22, 209 (1954).

[5] G. S. Rushbrooke, Proc. Roy. Soc. (London) [A] 166, 296 (1938).

Washington, May 23, 1957. 